\title{
Auto-titrating versus fixed continuous positive airway pressure for the treatment of obstructive sleep apnea: a systematic review with meta-analyses
}

Stanley Ip ${ }^{1}$, Carolyn D'Ambrosio ${ }^{1,2}$, Kamal Patel ${ }^{1}$, Ndidiamaka Obadan ${ }^{1}$, Georgios D Kitsios ${ }^{1}$, Mei Chung ${ }^{1}$ and Ethan M Balk ${ }^{1^{*}}$

\begin{abstract}
Background: Obstructive sleep apnea is a relatively common disorder that can lead to lost productivity and cardiovascular disease. The form of positive airway treatment that should be offered is unclear.

Methods: MEDLINE and the Cochrane Central Trials registry were searched for English language randomized controlled trials comparing auto-titrating positive airway pressure (APAP) with continuous positive airway pressure (CPAP) in adults with obstructive sleep apnea (inception through 9/2010). Six researchers extracted information on study design, potential bias, patient characteristics, interventions and outcomes. Data for each study were extracted by one reviewer and confirmed by another. Random effects model meta-analyses were performed for selected outcomes.

Results: Twenty-four randomized controlled trials met the inclusion criteria. In individual studies, APAP and fixed CPAP resulted in similar changes from baseline in the apnea-hypopnea index, most other sleep study measures and quality of life. By meta-analysis, APAP improved compliance by 11 minutes per night (95\% Cl, 3 to 19 minutes) and reduced sleepiness as measured by the Epworth Sleepiness Scale by 0.5 points ( $95 \%$ Cl, 0.8 to 0.2 point reduction) compared with fixed CPAP. Fixed CPAP improved minimum oxygen saturation by $1.3 \%$ more than APAP ( $95 \% \mathrm{Cl}, 0.4$ to $2.2 \%)$. Studies had relatively short follow-up and generally excluded patients with significant comorbidities. No study reported on objective clinical outcomes.

Conclusions: Statistically significant differences were found but clinical importance is unclear. Because the treatment effects are similar between APAP and CPAP, the therapy of choice may depend on other factors such as patient preference, specific reasons for non-compliance and cost.
\end{abstract}

\section{Background}

Obstructive sleep apnea (OSA) is a relatively common disorder in the US. The Wisconsin Sleep Cohort Study, a prospective natural history study, reported that about $10 \%$ of adults aged 30 to 60 years old had clear evidence of OSA in 1988, when the study began [1]. A National Sleep Foundation poll in 2005 suggested that as many

\footnotetext{
* Correspondence: ebalk@tuftsmedicalcenter.org 'Institute of Clinical Research and Health Policy Studies, Tufts University School of Medicine, Tufts Medical Center, Boston, MA, USA Full list of author information is available at the end of the article
}

as one in four American adults are at high risk of OSA and could benefit from an evaluation for OSA [2].

The defining characteristic of OSA is a partial or complete obstruction of the airway while sleeping. The most common first-line therapy is the use of continuous positive airway pressure (CPAP) devices during sleep. The CPAP machine directly relieves the airway obstruction by increasing luminal pressure, thereby splinting the airway open. When used properly and consistently, CPAP results in improved sleep patterns and quality of life due to decreased daytime somnolence. However, many patients refuse the offer of CPAP therapy, do not

\section{() Biomed Central}

(c) 2012 Ip et al; licensee BioMed Central Ltd. This is an Open Access article distributed under the terms of the Creative Commons Attribution License (http://creativecommons.org/licenses/by/2.0), which permits unrestricted use, distribution, and reproduction in any medium, provided the original work is properly cited. 
tolerate it or fail to use CPAP devices properly [3]. Patients commonly did not fully comply with CPAP use, either using the device for only part of the night or only on some nights. This non-compliance has fueled the development of a number of technological solutions.

The most common variation on delivering positive airway pressure is the use of auto-titrating positive airway pressure (APAP) devices. Fixed CPAP provides continuous fixed pressure during the entire sleep period. In contrast, APAP varies the pressure delivered depending on changes in airflow resistance. Such changes in airflow resistance during sleep are dependent on many factors like posture and the degree of nasal congestion. Theoretically, varying the pressure delivered would promote an increase in breathing synchrony with the CPAP device and therefore could improve patient comfort with the device and thus enhance compliance. A 2009 Cochrane review comparing APAP with CPAP concluded that APAP was slightly more efficacious than CPAP in increasing patient compliance [4]. We undertook the present review in the context of a larger review of all primary studies of treatments for OSA. We sought to update and expand upon the previous review. The aim of the present review is to evaluate the body of evidence regarding the comparative efficacy of APAP versus CPAP on clinical and sleep-related outcomes, quality of life, compliance and other outcomes.

\section{Methods}

We followed standard systematic review methods as described in the Agency for Healthcare Research and Quality (AHRQ) Methods Reference Guide for Effectiveness and Comparative Effectiveness Reviews [5]. A full technical report describing these methods in detail, including literature search strategies, and presenting our findings in full (with evidence tables) is available elsewhere [6].

We searched the MEDLINE and Cochrane Central Trials Registry databases from study inception to September 2010 for English language studies examining adults (older than 16 years) with OSA. Our search, available in the full technical report [6], included terms for OSA, sleep apnea treatments and relevant research designs. The full literature search was performed for a range of key questions about OSA diagnosis, treatment with any intervention and predictors of outcomes. Six reviewers independently screened the abstracts. We used a computerized screening program, abstrackr, to automate the screening of abstracts for the selection of eligible articles for full-text screening [7]. The abstrackr software uses an active learning algorithm to screen for relevant articles. Relevance was established by manually double-screening 1,000 abstracts to train the program. Subsequently, abstracts selected by the program were screened by one researcher. The results of screening were iteratively fed into the program for further training. This process continued until the program was left with only abstracts it rejected. Using abstrackr, we reduced by $50 \%$ the number of abstracts we needed to manually screen prior to starting the subsequent steps of the systematic review. Later, all abstracts rejected by abstrackr were manually screened for confirmation and were eventually rejected. Full-text articles were rescreened for eligibility by the same six reviewers.

We included peer reviewed, randomized controlled trials (RCTs) that compared APAP with fixed CPAP in $\geq 10$ patients per intervention with confirmed diagnoses of OSA, including a formal sleep study demonstrating an apnea-hypopnea index (AHI) $\geq 5$ events/hour. We included studies of any duration, though CPAP had to be used by the patients at home. Outcomes of interest included: objective clinical outcomes (death, cardiovascular events, hypertension, non-insulin dependent diabetes, depression); sleep and wakefulness related clinical outcomes (quality of life, sleepiness measures, neurocognitive tests, accidents, productivity); sleep study measures (AHI, arousal index, deep sleep, sleep efficiency, minimum oxygen saturation); comorbidity intermediate outcomes (hemoglobin A1c, blood pressure); compliance; and adverse events or harms.

Data from each study were extracted by one of six reviewers and confirmed by another. Extracted data included information on study and patient characteristics, details concerning the CPAP devices used, outcomes and study quality. For most outcomes, only data from the last reported time-point were included. We assessed the methodological quality of each study on the basis of predefined criteria in accordance with AHRQ's suggested methods for systematic reviews [5]. The primary data extractor determined the study quality (rated with the letter grades A, B or C), and at least one other reviewer confirmed it. Quality A studies adhered most closely to the commonly held precepts of high quality, including clear descriptions of the population, setting, interventions, outcomes and design; no obvious reporting omissions or errors; fewer than $20 \%$ dropouts; and no obvious source of bias. Quality B studies had some deficiencies in these criteria that were, however, unlikely to engender a major bias. Quality $\mathrm{C}$ studies had inadequate descriptions of their studies or had substantial flaws in reporting or design, such that a major bias could not be excluded.

We performed random effects model meta-analyses of differences of selected continuous variables between interventions where there were at least three unique similar studies [8]. Based on available data and our $a$ priori assessment of the clinical importance of specific outcomes, we performed meta-analyses for the AHI, the 
Epworth Sleepiness Scale (ESS), arousal index (per hour frequency of arousals from sleep), minimum oxygen saturation (during sleep), the multiple sleep latency test (measurement of how quickly a subject will fall asleep during the day), the quality of life measure Functional Outcomes Sleep Questionnaire and compliance (measured as time per night using the device). When necessary, standard errors of the net change (difference between the within-arm changes) were calculated from CIs, $P$ values or from the standard errors of the withinarm changes. When necessary, standard errors of the within-arm changes were estimated from the standard errors of the baseline and final values, assuming a 50\% correlation between the two. Studies that compared two different forms of APAP to CPAP were treated as independent despite the common CPAP arm. Due to limitations of the reported data and for consistency, in crossover studies we treated the difference in final values as equivalent to the net change, under the assumption that the baseline values were equal and would thus cancel out. Heterogeneity among effect sizes was assessed using the $\mathrm{I}^{2}$ index, and the chi-square test. An $\mathrm{I}^{2}$ index $\geq 50 \%$ was used to indicate medium-to-high heterogeneity [9].

To explore sources of heterogeneity in between-study findings, all forest plots were drawn with subgroup meta-analyses of trials stratified by baseline OSA severity (as determined by the minimum AHI threshold required in each study for the diagnosis of OSA). Forest plots sub-divided by study design are presented in the full technical report [6]. The decision to subgroup studies by minimum AHI and by study design was made $a$ priori; however, the minimum AHI categories were based on thresholds reported in the studies. We performed meta-regressions separately with AHI thresholds and study design to determine statistically significant differences among subgroups.

We graded the strength of the body of evidence based on the AHRQ Methods Reference Guide [5]. We took into account the overall study quality, the consistency across studies, the applicability of the studies to the general population of patients treated for OSA, the magnitude and precision of the treatment effects and the relative clinical importance of the different outcomes assessed [6]. The overall strength of evidence was rated as high, moderate, or low - which each indicate the level of confidence that the evidence reflects the true effect - or insufficient.

\section{Results}

Our literature search yielded 15,816 citations, from which 861 articles were retrieved (Figure 1). We identified $24 \mathrm{RCTs}$ that compared APAP with fixed CPAP treatment in patients with OSA (Table 1; [10-33]). Three RCTs [31-33] identified in prior meta-analyses $[4,34]$ were added after completion of our full technical

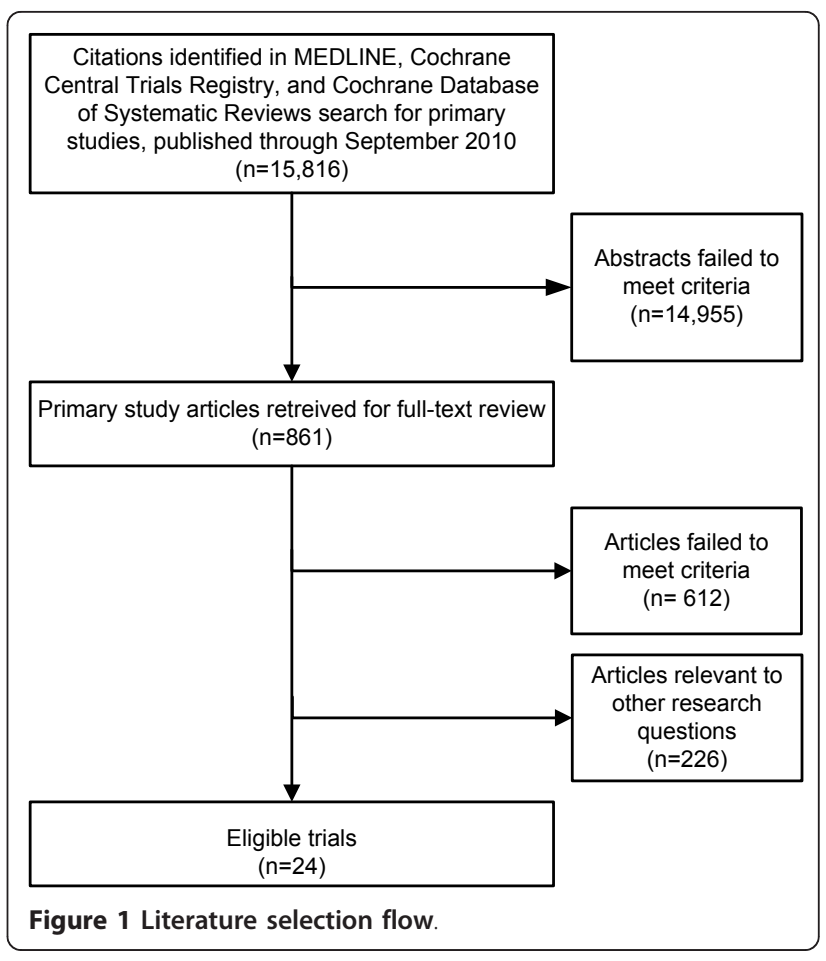

report [6]. Fifteen trials used a cross-over design and nine a parallel design. Studies generally failed to report complete data about outcomes. For 17 studies, the variance of the difference in baseline and final values was not reported and had to be estimated by making an assumption about the correlation between the values. Patients who were new to positive airway pressure treatments were enrolled in 21 of 24 studies (three did not provide this information). There was a broad range of OSA severity at baseline across studies; patients' mean baseline AHI ranged from 15 to 58 events/hour. In all studies, most patients were either overweight or obese (body mass index ranged from 29.9 to $42 \mathrm{~kg} / \mathrm{m}^{2}$ ). None of the studies selectively focused on patients with other comorbidities. Study sample sizes ranged from 10 to 181 patients (total 1017 across studies). Study durations ranged from three weeks to nine months, with the majority of studies lasting three months or less. Two trials were rated quality $A, 12$ were rated quality $B$ and ten rated quality C. Primary methodological concerns included small sample sizes without statistical power calculations, incomplete data reporting, short follow-up durations and high dropout rates. Based primarily on the eligibility criteria and baseline characteristics of the trial, the outcomes are applicable mainly to newly diagnosed (previously untreated) OSA patients with AHI > 15 events/ hour and body mass index $>30 \mathrm{~kg} / \mathrm{m}^{2}$. Outcome-specific tables summarizing the trials and their results can be found Table 2 (compliance), Table 3 (AHI), Table 4 (ESS), Table 5 (arousal index), Table 6 (minimum $\mathrm{O}_{2}$ 
Table 1 Randomized controlled trials of APAP versus CPAP: baseline study characteristics

\begin{tabular}{|c|c|c|c|c|c|c|c|c|}
\hline $\begin{array}{l}\text { Study } \\
\text { PMID }\end{array}$ & $\begin{array}{l}\text { Study } \\
\text { design }\end{array}$ & $\begin{array}{l}\text { Mean } \\
\text { age } \\
\text { (years) }\end{array}$ & $\begin{array}{l}\text { Male, } \\
\%\end{array}$ & $\begin{array}{l}\text { Mean body } \\
\text { mass index } \\
\left(\mathrm{kg} / \mathrm{m}^{2}\right)\end{array}$ & $\begin{array}{l}\text { Baseline } \\
\text { AHI (SD) }\end{array}$ & $\begin{array}{l}\text { Previous } \\
\text { CPAP? }\end{array}$ & Funding & Quality issues \\
\hline $\begin{array}{l}\text { d'Ortho } 2000[32] \\
11035671\end{array}$ & $\mathrm{XO}$ & 57 & 88 & 32 & $58(6)$ & No & $\begin{array}{l}\text { Industry/Non- } \\
\text { industry } \\
\text { combined }\end{array}$ & - \\
\hline $\begin{array}{l}\text { Damjanovic } 2009 \text { [10] } \\
19129293\end{array}$ & $P L$ & 57 & 78 & 31.1 & $44(25)$ & $\begin{array}{l}\text { No } \\
\text { (new Dx) }\end{array}$ & nd & - \\
\hline $\begin{array}{l}\text { Fietze } 2007[11] \\
17337881\end{array}$ & $P L$ & 54 & 95 & 30.9 & $42(26)$ & $\begin{array}{l}\text { No } \\
\text { (new Dx) }\end{array}$ & Industry & $\begin{array}{l}\text { Incomplete reporting; patient } \\
\text { selection unclear }\end{array}$ \\
\hline $\begin{array}{l}\text { Galetke } 2008[12] \\
17148931\end{array}$ & $\mathrm{XO}$ & 56 & 80 & 29.3 & $33(19)$ & $\begin{array}{l}\text { No } \\
\text { (new Dx) }\end{array}$ & nd & $\begin{array}{l}\text { Incomplete reporting; small sample } \\
\text { and no power calculation }\end{array}$ \\
\hline $\begin{array}{l}\text { Hudgel } 2000[18] \\
10947032\end{array}$ & XO & 46 & 54 & 42 & $30(25)$ & No & nd & Incomplete reporting; 35\% dropout \\
\hline $\begin{array}{l}\text { Hukins } 2004[19] \\
15683142\end{array}$ & XO & 50 & 87 & 35.2 & $50(25)$ & No & Industry & - \\
\hline $\begin{array}{l}\text { Hussain } 2004[20] \\
15072173\end{array}$ & $\mathrm{XO}$ & 45 & 90 & 35.9 & $47(36)$ & No & Industry & $\begin{array}{l}\text { Patient recruitment method unclear; } \\
\text { small sample and no power } \\
\text { calculation }\end{array}$ \\
\hline $\begin{array}{l}\text { Konermann } 1998[31] \\
9515848\end{array}$ & PL & 54 & 88 & 32.1 & $38(14)$ & nd & nd & - \\
\hline $\begin{array}{l}\text { Marrone } 2004[21] \\
15165530\end{array}$ & $\mathrm{XO}$ & 53 & 95 & 32.9 & $68(12)$ & No & nd & $\begin{array}{l}\text { Small sample and no power } \\
\text { calculation }\end{array}$ \\
\hline $\begin{array}{l}\text { Massie } 2003[22] \\
12406840\end{array}$ & $\mathrm{XO}$ & 49 & 82 & 32 & nd & $\begin{array}{l}\text { No } \\
\text { (new Dx, } \\
\text { implied) }\end{array}$ & Industry & Incomplete reporting \\
\hline $\begin{array}{l}\text { Meurice } 2007[13] \\
17638595\end{array}$ & PL & 55 & nd & 30.8 & $55(10)$ & $\begin{array}{l}\text { No } \\
\text { (no } T x)\end{array}$ & nd & Patient recruitment unclear \\
\hline $\begin{array}{l}\text { Nolan } 2007[14] \\
17326544\end{array}$ & XO & 53 & 90 & 29.9 & $15(8)$ & $\begin{array}{l}\text { No } \\
\text { (new Dx) }\end{array}$ & Non-industry & - \\
\hline $\begin{array}{l}\text { Noseda } 2004[23] \\
15249439\end{array}$ & XO & 49 & 96 & 32.3 & nd & No & nd & - \\
\hline $\begin{array}{l}\text { Nussbaumer } 2006[15] \\
16537862\end{array}$ & $\mathrm{XO}$ & 49 & 90 & 31.1 & $41(20)$ & nd & Industry & - \\
\hline $\begin{array}{l}\text { Patruno } 2007[16] \\
17494789\end{array}$ & $P L$ & 48 & 81 & 36.5 & $46(14)$ & $\begin{array}{l}\text { No } \\
\text { (new Dx) }\end{array}$ & Non-industry & Incomplete reporting \\
\hline $\begin{array}{l}\text { Planes } 2003[29] \\
12683473\end{array}$ & $P L$ & 54 & 77 & 32.4 & $59(17)$ & $\begin{array}{l}\text { No } \\
\text { (new Dx) }\end{array}$ & $\begin{array}{l}\text { Industry } \\
\text { (unclear) }\end{array}$ & Patient recruitment unclear \\
\hline $\begin{array}{l}\text { Randerath } 2001 \text { [26] } \\
11254519\end{array}$ & $\mathrm{XO}$ & 55 & 87 & 32.4 & $35(26)$ & nd & Industry & - \\
\hline $\begin{array}{l}\text { Resta } 2004[24] \\
15679008\end{array}$ & PL & 33 & 90 & 36.7 & $47(11)$ & $\begin{array}{l}\text { No } \\
\text { (no } T x)\end{array}$ & nd & Incomplete reporting \\
\hline $\begin{array}{l}\text { Senn } 2003[25] \\
14525804\end{array}$ & $\mathrm{XO}$ & 53 & 79 & 33.3 & $46(23)$ & No & nd & - \\
\hline $\begin{array}{l}\text { Sériès } 1997 \text { [27] } \\
9341056\end{array}$ & PL & $\begin{array}{l}36-65 \\
\text { (range) }\end{array}$ & nd & 36.4 & $44(20)$ & $\begin{array}{l}\text { No } \\
\text { (no Tx) }\end{array}$ & Industry & Potential selection bias \\
\hline $\begin{array}{l}\text { Teschler } 2000[28] \\
10885414\end{array}$ & XO & 52 & 100 & 33.8 & $53(26)$ & $\begin{array}{l}\text { No } \\
\text { (new Dx) }\end{array}$ & nd & Incomplete reporting; small sample \\
\hline $\begin{array}{l}\text { To } 2008 \text { [17] } \\
18197915\end{array}$ & $\mathrm{XO}$ & 46 & nd & 28.7 & 54 (nd) & $\begin{array}{l}\text { No } \\
\text { (new Dx) }\end{array}$ & nd & - \\
\hline $\begin{array}{l}\text { Vennelle } 2010 \text { [30] } \\
20175411\end{array}$ & $\mathrm{XO}$ & 50 & 77 & 34.5 & $33(18)$ & $\begin{array}{l}\text { No } \\
\text { (new Dx) }\end{array}$ & nd & - \\
\hline $\begin{array}{l}\text { West } 2006 \text { [33] } \\
16254055\end{array}$ & $\mathrm{PL}$ & $46.5^{\mathrm{a}}$ & 85 & nd & nd & No & Industry & - \\
\hline
\end{tabular}

APAP: auto-titrating CPAP; CPAP: continuous positive airway pressure; nd: no data; new Dx: a new diagnosis of sleep apnea; no Tx: no prior treatment; PL: parallel; XO: cross-over. ${ }^{a}$ Median of the CPAP arm. 
Table 2 Compliance (mean hours/night) in randomized controlled trials of APAP versus CPAP

\begin{tabular}{|c|c|c|c|c|c|c|c|c|c|c|c|}
\hline $\begin{array}{l}\text { Study } \\
\text { PMID }\end{array}$ & $\begin{array}{l}\text { Baseline } \\
\text { AHI (SD) } \\
\text { [Eligibility] }\end{array}$ & $\begin{array}{l}\text { Baseline } \\
\text { ESS (SD) }\end{array}$ & $\begin{array}{l}\text { Duration } \\
\text { (design) }\end{array}$ & Interventions & $\begin{array}{l}\text { Number } \\
\text { analyzed }\end{array}$ & Final & $\begin{array}{l}\text { Change } \\
\text { (final) }\end{array}$ & $\begin{array}{l}\text { Net } \\
\text { difference } \\
\text { or } \\
\text { difference }\end{array}$ & $P$ & $\begin{array}{l}\text { Dropout } \\
\text { (\%) }\end{array}$ & $\begin{array}{l}\text { Study } \\
\text { quality }\end{array}$ \\
\hline \multirow[t]{2}{*}{$\begin{array}{l}\text { d'Ortho } 2000 \text { [32] } \\
11035671\end{array}$} & $\begin{array}{l}58(6) \\
{[\geq 10]}\end{array}$ & $12.7(5.3)$ & $\begin{array}{l}2 \text { months } \\
(X O)\end{array}$ & APAP & 25 & 4.1 & -0.6 & $-1.5,0.32^{a}$ & 0.20 & 0 & B \\
\hline & & & & CPAP & 25 & 4.7 & & & & & \\
\hline \multirow{4}{*}{$\begin{array}{l}\text { Damjanovic } 2009 \\
\text { [10] } \\
19129293\end{array}$} & $\begin{array}{l}44(25) \\
{[\geq 15]}\end{array}$ & $8.8(5.2)$ & $\begin{array}{l}3 \text { months } \\
(\mathrm{PL})\end{array}$ & APAP & 46 & 5.4 & 0 & $-0.7,0.7$ & nd & 8 & B \\
\hline & & & & CPAP & 46 & 5.4 & & & & & \\
\hline & & & $\begin{array}{l}9 \text { months } \\
(\mathrm{PL})\end{array}$ & APAP & 34 & 5.2 & 0.1 & $-0.9,1.1$ & nd & 22 & \\
\hline & & & & CPAP & 44 & 5.1 & & & & & \\
\hline \multirow[t]{2}{*}{$\begin{array}{l}\text { Fietze } 2007[11] \\
17337881\end{array}$} & $\begin{array}{l}42(26) \\
{[\geq 10]}\end{array}$ & nd & $\begin{array}{l}1.5 \text { months } \\
(\mathrm{PL})\end{array}$ & APAP & 20 & 5.0 & 0.8 & nd & NS & 0 & C \\
\hline & & & & CPAP & 21 & 4.2 & & & & & \\
\hline \multirow[t]{2}{*}{$\begin{array}{l}\text { Galetke } 2008 \text { [12] } \\
17148931\end{array}$} & $\begin{array}{l}33(19) \\
{[>10]}\end{array}$ & $10.3(5.7)$ & $\begin{array}{l}2 \text { months } \\
(X O)\end{array}$ & APAP & 20 & 6.37 & -0.01 & $-0.82,0.8$ & nd & nd & C \\
\hline & & & & CPAP & 20 & 6.38 & & & & & \\
\hline \multirow[t]{2}{*}{$\begin{array}{l}\text { Hudgel } 2000 \text { [18] } \\
10947032\end{array}$} & $\begin{array}{l}30(25) \\
{[n d]}\end{array}$ & $16.0(5.0)$ & $\begin{array}{l}3 \text { months } \\
(X O)\end{array}$ & APAP & 14 & 6.0 & 0.5 & $0.02,0.98^{\mathrm{a}}$ & $<0.04$ & 35 & C \\
\hline & & & & CPAP & 19 & 5.5 & & & & & \\
\hline \multirow[t]{2}{*}{$\begin{array}{l}\text { Hukins } 2004 \text { [19] } \\
15683142\end{array}$} & 56 (nd) & 12.5 (nd) & $\begin{array}{l}1 \text { to } 2 \\
\text { months } \\
(\mathrm{XO})\end{array}$ & APAP & 46 & 5.05 & 0.19 & $-0.06,0.44^{\mathrm{a}}$ & 0.14 & 16 & B \\
\hline & & & & CPAP & 46 & 4.86 & & & & & \\
\hline \multirow[t]{2}{*}{$\begin{array}{l}\text { Hussain } 2004 \text { [20] } \\
15072173\end{array}$} & $\begin{array}{l}47(36) \\
{[>15]}\end{array}$ & $11.1(6.4)$ & $\begin{array}{l}1 \text { month } \\
(\mathrm{XO})\end{array}$ & APAP & 10 & 4.3 & 0.6 & $-0.84,2.04$ & nd & 0 & C \\
\hline & & & & CPAP & 10 & 3.7 & & & & & \\
\hline \multirow[t]{2}{*}{$\begin{array}{l}\text { Konermann } 1998 \\
\text { [31] } \\
9515848\end{array}$} & $\begin{array}{l}38(14) \\
{[>20]}\end{array}$ & nd & $\begin{array}{l}3 \text { to } 6 \\
\text { months } \\
\text { (PL) }\end{array}$ & APAP & 25 & 6.5 & 0.8 & $0.19,1.41^{\mathrm{a}}$ & $<0.01$ & 4 & B \\
\hline & & & & CPAP & 23 & 5.7 & & & & & \\
\hline \multirow[t]{2}{*}{$\begin{array}{l}\text { Marrone } 2004 \text { [21] } \\
15165530\end{array}$} & $\begin{array}{l}68(12) \\
{[30]}\end{array}$ & $16.3(5.0)$ & $\begin{array}{l}1 \text { month } \\
(X O)\end{array}$ & APAP & 22 & 4.9 & 0.5 & $-0.26,1.26$ & nd & 0 & C \\
\hline & & & & CPAP & 22 & 4.4 & & & & & \\
\hline \multirow[t]{2}{*}{$\begin{array}{l}\text { Massie } 2003[22] \\
12406840\end{array}$} & $\begin{array}{l}\text { nd } \\
{[\geq 15]}\end{array}$ & nd & $\begin{array}{l}1.5 \text { months } \\
(\mathrm{XO})\end{array}$ & APAP & 44 & 5.1 & 0.58 & $0.18,0.99^{\mathrm{a}}$ & 0.005 & 4 & B \\
\hline & & & & CPAP & 44 & 4.52 & & & & & \\
\hline \multirow[t]{4}{*}{$\begin{array}{l}\text { Meurice } 2007 \text { [13] } \\
17638595\end{array}$} & $\begin{array}{l}55(10) \\
{[\mathrm{nd}]}\end{array}$ & $11.8(4.9)$ & $\begin{array}{l}3 \text { months } \\
(\mathrm{PL})\end{array}$ & $\begin{array}{l}\text { APAP } \\
\text { (AutoSet) }\end{array}$ & 15 & 6.0 & -0.1 & $-0.79,0.59$ & nd & 15 & B \\
\hline & & & & CPAP & 14 & 6.1 & & & & & \\
\hline & & & $\begin{array}{l}6 \text { months } \\
(\mathrm{PL})\end{array}$ & $\begin{array}{l}\text { APAP } \\
\text { (AutoSet) }\end{array}$ & 15 & 6.1 & -0.4 & $-1.28,0.48$ & nd & 15 & \\
\hline & & & & CPAP & 14 & 6.5 & & & & & \\
\hline \multirow[t]{2}{*}{$\begin{array}{l}\text { Nolan } 2007 \text { [14] } \\
17326544\end{array}$} & $\begin{array}{l}15(8) \\
{[\geq 5]}\end{array}$ & $12.3(4.0)$ & $\begin{array}{l}2 \text { months } \\
(X O)\end{array}$ & APAP & 29 & 4.9 & 0 & nd & 0.94 & 15 & B \\
\hline & & & & CPAP & 29 & 4.9 & & & & & \\
\hline \multirow[t]{2}{*}{$\begin{array}{l}\text { Noseda } 2004 \text { [23] } \\
15249439\end{array}$} & $\begin{array}{l}51(25)[> \\
20]\end{array}$ & $10.7(2.4)$ & $\begin{array}{l}2 \text { months } \\
(X O)\end{array}$ & APAP & 24 & 5.3 & -0.2 & $-0.89,0.49$ & nd & 11 & B \\
\hline & & & & CPAP & 24 & 5.5 & & & & & \\
\hline \multirow{2}{*}{$\begin{array}{l}\text { Nussbaumer } 2006 \\
\text { [15] } \\
16537862\end{array}$} & $\begin{array}{l}41(20) \\
{[>10]}\end{array}$ & 12.7 (3.3) & $\begin{array}{l}1 \text { month } \\
(\mathrm{XO})\end{array}$ & APAP & 30 & 5.1 & 0.3 & $-0.29,0.89$ & nd & 12 & B \\
\hline & & & & CPAP & 30 & 4.8 & & & & & \\
\hline $\begin{array}{l}\text { Patruno } 2007 \text { [16] } \\
17494789\end{array}$ & $\begin{array}{l}46(14) \\
{[>20]}\end{array}$ & $15(2.7)$ & $\begin{array}{l}3 \text { months } \\
(\mathrm{PL})\end{array}$ & APAP & 15 & 6.2 & 0.2 & $-0.25,0.65$ & nd & 23 & C \\
\hline
\end{tabular}


Table 2 Compliance (mean hours/night) in randomized controlled trials of APAP versus CPAP (Continued)

\begin{tabular}{|c|c|c|c|c|c|c|c|c|c|c|c|}
\hline & & & & CPAP & 16 & 6.0 & & & & & \\
\hline \multirow[t]{2}{*}{$\begin{array}{l}\text { Planes } 2003 \text { [29] } \\
12683473\end{array}$} & $\begin{array}{l}59(17) \\
{[\geq 30]}\end{array}$ & $15.1(3.9)$ & $\begin{array}{l}2 \text { months } \\
(P L)\end{array}$ & APAP & 16 & 4.5 & -0.8 & nd & NS & 14 & C \\
\hline & & & & CPAP & 14 & 5.3 & & & & & \\
\hline \multirow{2}{*}{$\begin{array}{l}\text { Randerath } 2001 \\
\text { [26] } \\
11254519\end{array}$} & $\begin{array}{l}35(26) \\
{[\geq 10]}\end{array}$ & $11.1(5.1)$ & $\begin{array}{l}1.5 \text { months } \\
(X O)\end{array}$ & APAP & 46 & 5.26 & 0 & $-0.44,0.44$ & nd & 12 & B \\
\hline & & & & CPAP & 46 & 5.26 & & & & & \\
\hline \multirow[t]{2}{*}{$\begin{array}{l}\text { Resta } 2004 \text { [24] } \\
15679008\end{array}$} & $\begin{array}{l}47(11) \\
{[>30]}\end{array}$ & 13.9 (3.2) & $\begin{array}{l}1 \text { month } \\
(\mathrm{PL})\end{array}$ & APAP & 10 & 5.2 & -0.1 & $-1.12,0.92$ & nd & 0 & C \\
\hline & & & & CPAP & 10 & 5.3 & & & & & \\
\hline \multirow[t]{3}{*}{$\begin{array}{l}\text { Senn } 2003 \text { [25] } \\
14525804\end{array}$} & $\begin{array}{l}46(23) \\
{[>10]}\end{array}$ & 14.2 (3.8) & $\begin{array}{l}1 \text { month } \\
(\mathrm{XO})\end{array}$ & $\begin{array}{l}\text { APAP } \\
\text { (Autoset T) }\end{array}$ & 29 & 5.5 & -0.1 & nd & NS & 7 & B \\
\hline & & & & $\begin{array}{l}\text { APAP } \\
\text { (AutoAdjust) }\end{array}$ & 29 & 5.5 & -0.1 & nd & NS & & \\
\hline & & & & CPAP & 29 & 5.6 & & & & & \\
\hline \multirow[t]{4}{*}{$\begin{array}{l}\text { Sériès } 1997 \text { [27] } \\
9341056\end{array}$} & $\begin{array}{l}44(20) \\
{[n d]}\end{array}$ & $15.5(4.5)$ & $\begin{array}{l}0.75 \\
\text { months } \\
(\mathrm{PL})\end{array}$ & $\mathrm{APAP}^{\mathrm{b}}$ & 12 & $\mathrm{nd}^{\mathrm{c}}$ & - & - & NS & 0 & C \\
\hline & & & & CPAP & 12 & nd & & & & & \\
\hline & & & & APAP $^{d}$ & 12 & $n d^{c}$ & - & - & NS & & \\
\hline & & & & CPAP & 12 & nd & & & & & \\
\hline \multirow{2}{*}{$\begin{array}{l}\text { Vennelle } 2010 \\
\text { [30] } \\
20175411\end{array}$} & $\begin{array}{l}33(18) \\
{[\geq 15]}\end{array}$ & $14(3)$ & $\begin{array}{l}6 \text { weeks } \\
(X O)\end{array}$ & APAP & 181 & 4.2 & 0.2 & $\begin{array}{l}0.003 \\
0.397\end{array}$ & 0.047 & 9.5 & A \\
\hline & & & & CPAP & 181 & 4.0 & & & & & \\
\hline \multirow[t]{2}{*}{$\begin{array}{l}\text { Teschler } 2000 \text { [28] } \\
10885414\end{array}$} & $\begin{array}{l}53(26) \\
{[>20]}\end{array}$ & nd & $\begin{array}{l}2 \text { months } \\
(X O)\end{array}$ & APAP & 10 & 6.3 & 0.2 & $-0.7,1.1$ & nd & nd & C \\
\hline & & & & CPAP & 10 & 6.1 & & & & & \\
\hline \multirow[t]{4}{*}{$\begin{array}{l}\text { To } 2008 \text { [17] } \\
18197915\end{array}$} & $\begin{array}{l}54 \text { (nd) } \\
{[>30]}\end{array}$ & 13.4 (nd) & $\begin{array}{l}1 \text { month } \\
(X O)\end{array}$ & APAP & 41 & 4.3 & 0.5 & $0.02,0.98$ & 0.04 & 5 & B \\
\hline & & & & CPAP & 41 & 3.8 & & & & & \\
\hline & & & $\begin{array}{l}2 \text { months } \\
(X O)\end{array}$ & APAP & 41 & 4.4 & 0.7 & $0.17,1.23^{a}$ & 0.01 & & \\
\hline & & & & CPAP & 41 & 3.7 & & & & & \\
\hline \multirow[t]{6}{*}{$\begin{array}{l}\text { West } 2006 \text { [33] } \\
16254055\end{array}$} & $\begin{array}{l}33^{e}(\mathrm{nd}) \\
{[>10]}\end{array}$ & $16.5^{f}(\mathrm{nd})$ & $\begin{array}{l}1 \text { month } \\
\text { (PL) }\end{array}$ & APAP & 29 & 5.3 & & & 0.27 & 6.1 & A \\
\hline & & & & CPAP (auto) & 31 & 4.3 & & & & & \\
\hline & & & & CPAP (algo) & 32 & 4.7 & & & & & \\
\hline & & & $\begin{array}{l}6 \text { months } \\
(\mathrm{PL})\end{array}$ & APAP & 28 & 5.5 & & & 0.23 & & \\
\hline & & & & CPAP (auto) & 31 & 4.9 & & & & & \\
\hline & & & & CPAP (algo) & 27 & 4.0 & & & & 9.2 & \\
\hline
\end{tabular}

AHI: apnea-hypopnea index (events/hour); algo: fixed pressure CPAP determined by algorithm; APAP: auto-titrating CPAP; auto: fixed pressure CPAP determined by pressures from 1 week of APAP; CPAP: continuous positive airway pressure; ESS: Epworth Sleepiness Scale; nd: no data; NS: non-significant; PL: parallel design; XO: cross-over design. ${ }^{a}$ Estimated from reported $P$ value. ${ }^{b}$ Estimated effective pressure. ${ }^{c}$ Directions of changes not reported in the study. ${ }^{\mathrm{d}} \mathrm{Measured}$ effective pressure. ${ }^{\mathrm{e}}>4 \% \mathrm{SaO}_{2}$ dip rate per hour in algorithm-determined CPAP arm, median. ${ }^{\mathrm{f}}$ Median in algorithm-determined CPAP arm.

saturation), Table 7 (sleep efficiency), Table 8 (rapid eye movement (REM) sleep), Table 9 (stage 3 or 4 sleep) and Table 10 (quality of life and functional outcomes).

\section{Objective clinical outcomes}

No trial evaluated clinical outcomes, including death; cardiovascular events such as myocardial infarction, heart failure or stroke; or diabetes or depression severity.

\section{Compliance}

All 24 included trials reported data on compliance. The number of hours used per night was derived from machine-recorded compliance data. No statistically significant differences were observed in device usage (hours used per night) between APAP and CPAP in 20 of the trials, while four reported a significant increase in the use of APAP compared with CPAP $[17,18,22,30]$. Twenty-two trials provided sufficient data for meta- 
Table $3 \mathrm{AHI}$ (events/hour) in randomized controlled trials of APAP versus CPAP

\begin{tabular}{|c|c|c|c|c|c|c|c|c|c|c|c|c|}
\hline $\begin{array}{l}\text { Study } \\
\text { PMID }\end{array}$ & $\begin{array}{l}\text { Baseline AHI } \\
\text { (SD) } \\
\text { [eligibility] }\end{array}$ & $\begin{array}{l}\text { Baseline ESS } \\
\text { (SD) }\end{array}$ & $\begin{array}{l}\text { Duration } \\
\text { (design) }\end{array}$ & Interventions & $\begin{array}{l}\text { Number } \\
\text { analyzed }\end{array}$ & $\begin{array}{l}\text { Baseline } \\
\text { (SD) }\end{array}$ & $\begin{array}{l}\text { Change } \\
\text { (final) }\end{array}$ & $\begin{array}{l}\text { Net } \\
\text { difference } \\
\text { or } \\
\text { difference }\end{array}$ & $95 \% \mathrm{Cl}^{\mathrm{a}}$ & $P$ & $\begin{array}{l}\text { Dropout } \\
\text { (\%) }\end{array}$ & $\begin{array}{l}\text { Study } \\
\text { quality }\end{array}$ \\
\hline \multirow[t]{2}{*}{$\begin{array}{l}\text { d'Ortho } 2000 \text { [32] } \\
11035671\end{array}$} & $\begin{array}{l}58(6) \\
{[\geq 10]}\end{array}$ & $12.7(5.3)$ & $\begin{array}{l}2 \text { months } \\
(X O)\end{array}$ & APAP & 25 & $57.8(5.8)$ & -47.2 & 0.9 & nd & NS & 0 & B \\
\hline & & & & CPAP & 25 & $\begin{array}{l}57.8 \\
(5.8)\end{array}$ & -48.1 & & & & & \\
\hline \multirow[t]{4}{*}{$\begin{array}{l}\text { Damjanovic } 2009 \text { [10] } \\
19129293\end{array}$} & $\begin{array}{l}44(25) \\
{[\geq 15]}\end{array}$ & $8.8(5.2)$ & $\begin{array}{l}3 \text { months } \\
(\mathrm{PL})\end{array}$ & APAP & 46 & $41.8(23.7)$ & -37.0 & 1.8 & $\begin{array}{l}-7.14 \text { to } \\
10.74\end{array}$ & nd & 8 & B \\
\hline & & & & CPAP & 46 & $45.5(24.4)$ & -38.8 & & & & & \\
\hline & & & $\begin{array}{l}9 \text { months } \\
(\mathrm{PL})\end{array}$ & APAP & 34 & $41.8(23.7)$ & -38.2 & 1.9 & $\begin{array}{l}-6.86 \text { to } \\
10.66\end{array}$ & nd & 22 & \\
\hline & & & & CPAP & 44 & $45.5(24.4)$ & -40.1 & & & & & \\
\hline \multirow[t]{2}{*}{$\begin{array}{l}\text { Fietze } 2007 \text { [11] } \\
17337881\end{array}$} & $\begin{array}{l}42(26) \\
{[\geq 10]}\end{array}$ & nd & $\begin{array}{l}1.5 \text { months } \\
(\mathrm{PL})\end{array}$ & APAP & 20 & $43.3(30.2)$ & -38.9 & 0.5 & -1.19 to 2.19 & nd & 0 & C \\
\hline & & & & CPAP & 21 & $40.4(26.1)$ & -36.5 & & & & & \\
\hline \multirow[t]{2}{*}{$\begin{array}{l}\text { Galetke } 2008 \text { [12] } \\
17148931\end{array}$} & $\begin{array}{l}33(19) \\
{[>10]}\end{array}$ & $10.3(5.7)$ & $\begin{array}{l}2 \text { months } \\
(X O)\end{array}$ & APAP & 20 & $32.9(19.1)$ & -27.3 & 1.0 & -0.45 to 2.45 & nd & nd & C \\
\hline & & & & CPAP & 20 & $32.9(19.1)$ & -28.3 & & & & & \\
\hline \multirow[t]{2}{*}{$\begin{array}{l}\text { Hussain } 2004 \text { [20] } \\
15072173\end{array}$} & $\begin{array}{l}47(36) \\
{[>15]}\end{array}$ & $11.1(6.4)$ & $\begin{array}{l}1 \text { month } \\
(\mathrm{XO})\end{array}$ & APAP & 10 & $47.2(35.6)$ & -34.1 & 3.5 & -1.02 to 8.02 & nd & 0 & C \\
\hline & & & & CPAP & 10 & $47.2(35.6)$ & -37.6 & & & & & \\
\hline \multirow[t]{2}{*}{$\begin{array}{l}\text { Konermann } 1998 \text { [31] } \\
9515848\end{array}$} & $\begin{array}{l}38(14) \\
{[>20]}\end{array}$ & nd & $\begin{array}{l}3 \text { to } 6 \text { months } \\
\text { (PL) }\end{array}$ & APAP & 25 & $\begin{array}{l}35.5 \\
(9.6)\end{array}$ & -33.1 & 1.6 & nd & NS & 4 & B \\
\hline & & & & CPAP & 23 & $\begin{array}{l}38.3 \\
(13.9)\end{array}$ & -34.7 & & & & & \\
\hline \multirow[t]{2}{*}{$\begin{array}{l}\text { Massie } 2003 \text { [22] } \\
12406840\end{array}$} & $\begin{array}{l}\text { nd } \\
{[\geq 15]}\end{array}$ & nd & $\begin{array}{l}1.5 \text { months } \\
(\mathrm{XO})\end{array}$ & APAP & 44 & nd & nd & -1.1 & -2.89 to 0.69 & nd & 4 & B \\
\hline & & & & CPAP & 44 & nd & nd & & & & & \\
\hline \multirow[t]{2}{*}{$\begin{array}{l}\text { Meurice } 2007 \text { [13] } \\
17638595\end{array}$} & $\begin{array}{l}55(10) \\
{[n d]}\end{array}$ & $11.8(4.9)$ & $\begin{array}{l}6 \text { months } \\
(\mathrm{PL})\end{array}$ & APAP (AutoSet) & 15 & $53.4(15.1)$ & -51.1 & 2.6 & $\begin{array}{l}-8.88 \text { to } \\
14.08\end{array}$ & nd & 15 & B \\
\hline & & & & CPAP & 14 & $\begin{array}{l}56.1 \\
(21.4)\end{array}$ & -53.7 & & & & & \\
\hline \multirow[t]{2}{*}{$\begin{array}{l}\text { Nolan } 2007 \text { [14] } \\
17326544\end{array}$} & $\begin{array}{l}15(8) \\
{[\geq 5)}\end{array}$ & $12.3(4.0)$ & $\begin{array}{l}2 \text { months } \\
(X O)\end{array}$ & APAP & 29 & 14.7 (8) & -12.0 & -0.8 & $\begin{array}{l}-1.89 \text { to } \\
0.29^{\mathrm{b}}\end{array}$ & 0.15 & 15 & B \\
\hline & & & & CPAP & 29 & 14.7 (8) & -11.2 & & & & & \\
\hline \multirow[t]{2}{*}{$\begin{array}{l}\text { Nussbaumer } 2006 \text { [15] } \\
16537862\end{array}$} & $\begin{array}{l}41(20) \\
{[>10]}\end{array}$ & 12.7 (3.3) & $\begin{array}{l}1 \text { month } \\
(\mathrm{XO})\end{array}$ & APAP & 30 & $41.1(19.7)$ & -36.5 & -0.8 & -1.7 to $3.3^{c}$ & nd & 12 & B \\
\hline & & & & CPAP & 30 & $41.2(19.7)$ & -35.7 & & & & & \\
\hline \multirow[t]{2}{*}{$\begin{array}{l}\text { Patruno } 2007 \text { [16] } \\
17494789\end{array}$} & $\begin{array}{l}46(14) \\
{[>20]}\end{array}$ & $15(2.7)$ & $\begin{array}{l}3 \text { months } \\
(\mathrm{PL})\end{array}$ & APAP & 15 & $47.3(14.7)$ & -41.3 & 2.7 & $\begin{array}{l}-7.01 \text { to } \\
12.41\end{array}$ & nd & 23 & $C$ \\
\hline & & & & CPAP & 16 & $\begin{array}{l}46.0 \\
(14.6)\end{array}$ & -44.0 & & & & & \\
\hline
\end{tabular}


Table $3 \mathrm{AHI}$ (events/hour) in randomized controlled trials of APAP versus CPAP (Continued)

\begin{tabular}{|c|c|c|c|c|c|c|c|c|c|c|c|c|}
\hline \multirow[t]{2}{*}{$\begin{array}{l}\text { Planes } 2003 \text { [29] } \\
12683473\end{array}$} & $\begin{array}{l}59(17) \\
{[\geq 30]}\end{array}$ & $15.1(3.9)$ & $\begin{array}{l}2 \text { months } \\
(\mathrm{PL})\end{array}$ & APAP & 16 & $57.5(16.5)$ & -49.9 & 0.7 & $\begin{array}{l}-10.06 \text { to } \\
11.46\end{array}$ & nd & 14 & C \\
\hline & & & & CPAP & 14 & $\begin{array}{l}61.0 \\
(17.4)\end{array}$ & -50.6 & & & & & \\
\hline \multirow[t]{2}{*}{$\begin{array}{l}\text { Randerath } 2001 \text { [26] } \\
11254519\end{array}$} & $\begin{array}{l}35(26) \\
{[\geq 10]}\end{array}$ & $11.1(5.1)$ & $\begin{array}{l}1.5 \text { months } \\
(\mathrm{XO})\end{array}$ & APAP & 52 & $35.1(26)$ & -30.1 & 0.7 & -0.88 to 2.28 & nd & 12 & B \\
\hline & & & & CPAP & 52 & $35.1(26)$ & -30.8 & & & & & \\
\hline \multirow[t]{2}{*}{$\begin{array}{l}\text { Resta } 2004 \text { [24] } \\
15679008\end{array}$} & $\begin{array}{l}47(11) \\
{[>30]}\end{array}$ & 13.9 (3.2) & $\begin{array}{l}1 \text { month } \\
(\mathrm{PL})\end{array}$ & APAP & 10 & $\begin{array}{l}48.0 \\
(14.3)\end{array}$ & -39.7 & -2.8 & $\begin{array}{l}-12.96 \text { to } \\
7.36\end{array}$ & nd & 0 & C \\
\hline & & & & CPAP & 10 & $45.3(10.7)$ & -36.9 & & & & & \\
\hline \multirow{3}{*}{$\begin{array}{l}\text { Senn } 2003 \text { [25] } \\
\text { Switzerland } \\
14525804\end{array}$} & $\begin{array}{l}46(23) \\
{[>10]}\end{array}$ & $14.2(3.8)$ & $\begin{array}{l}1 \text { month } \\
(\mathrm{XO})\end{array}$ & $\begin{array}{l}\text { APAP (Autoset } \\
\text { T) }\end{array}$ & 29 & $45.8(22.6)$ & -39.8 & 0.7 & -1.26 to 2.66 & nd & 7 & B \\
\hline & & & & $\begin{array}{l}\text { APAP } \\
\text { (AutoAdjust) }\end{array}$ & 29 & $45.8(22.6)$ & -38.1 & 2.4 & -0.34 to 5.14 & nd & & \\
\hline & & & & CPAP & 29 & $\begin{array}{l}45.8 \\
(22.6)\end{array}$ & -40.5 & & & & & \\
\hline \multirow[t]{4}{*}{$\begin{array}{l}\text { Sériès } 1997 \text { [27] } \\
9341056\end{array}$} & $\begin{array}{l}44(20) \\
\text { [nd] }\end{array}$ & $15.5(4.5)$ & $\begin{array}{l}0.75 \text { months } \\
(\mathrm{PL})\end{array}$ & APAPd $^{d}$ & 12 & $61.5(27.9)$ & $n d^{e}$ & - & - & NS & 0 & C \\
\hline & & & & CPAP & 12 & $50.1(14.5)$ & nd & & & & & \\
\hline & & & & APAP $^{f}$ & 12 & $46.8(22.3)$ & $\mathrm{nd}^{\mathrm{e}}$ & - & - & NS & & \\
\hline & & & & CPAP & 12 & $50.1(14.5)$ & nd & & & & & \\
\hline
\end{tabular}

AHI: apnea-hypopnea index (events/hour); APAP: auto-titrating CPAP; CPAP: continuous positive airway pressure; ESS: Epworth Sleepiness Scale; nd: no data; NS: non-significant; PL: parallel design; XO: cross-over design. ${ }^{a}$ Estimated from reported data. ${ }^{b}$ Estimated from reported $P$ value. ${ }^{c}$ Actual reported data. ${ }^{d}$ Estimated effective pressure. ${ }^{e}$ Directions of changes not reported in the study. ${ }^{f}$ Measured effective pressure. 
Table 4 ESS in randomized controlled trials of APAP versus CPAP

\begin{tabular}{|c|c|c|c|c|c|c|c|c|c|c|c|c|}
\hline $\begin{array}{l}\text { Study } \\
\text { PMID }\end{array}$ & $\begin{array}{l}\text { Baseline AHI } \\
\text { (SD) } \\
\text { [eligibility] }\end{array}$ & $\begin{array}{l}\text { Baseline ESS } \\
\text { (SD) }\end{array}$ & $\begin{array}{l}\text { Duration } \\
\text { (design) }\end{array}$ & Interventions & $\begin{array}{l}\text { Number } \\
\text { analyzed }\end{array}$ & $\begin{array}{l}\text { Baseline } \\
\text { (SD) }\end{array}$ & $\begin{array}{l}\text { Change } \\
\text { (final) }\end{array}$ & $\begin{array}{l}\text { Net } \\
\text { difference } \\
\text { or } \\
\text { difference }\end{array}$ & $95 \% \mathrm{Cl}^{\mathrm{a}}$ & $P$ & $\begin{array}{l}\text { Dropout } \\
\text { (\%) }\end{array}$ & $\begin{array}{l}\text { Study } \\
\text { quality }\end{array}$ \\
\hline \multirow[t]{2}{*}{$\begin{array}{l}\text { d'Ortho } 2000 \text { [32] } \\
11035671\end{array}$} & $\begin{array}{l}58(6) \\
{[\geq 10]}\end{array}$ & $12.7(5.3)$ & $\begin{array}{l}2 \text { months } \\
(\mathrm{XO})\end{array}$ & APAP & 25 & $12.7(5.3)$ & -3.4 & 0.1 & nd & NS & 0 & B \\
\hline & & & & CPAP & 25 & $\begin{array}{l}12.7 \\
(5.3)\end{array}$ & -3.5 & & & & & \\
\hline \multirow[t]{4}{*}{$\begin{array}{l}\text { Damjanovic } 2009 \text { [10] } \\
19129293\end{array}$} & $\begin{array}{l}44(25) \\
{[\geq 15]}\end{array}$ & $8.8(5.2)$ & $\begin{array}{l}3 \text { months } \\
(\mathrm{PL})\end{array}$ & APAP & 46 & $8.5(5.4)$ & -2.6 & -0.3 & $\begin{array}{l}-2.32 \text { to } \\
1.72\end{array}$ & nd & 8 & B \\
\hline & & & & CPAP & 46 & $9.3(4.8)$ & -2.3 & & & & & \\
\hline & & & $\begin{array}{l}9 \text { months } \\
(\mathrm{PL})\end{array}$ & APAP & 34 & $8.5(5.4)$ & -2.6 & 0.1 & $\begin{array}{l}-1.92 \text { to } \\
2.12\end{array}$ & nd & 22 & \\
\hline & & & & CPAP & 44 & $9.3(4.8)$ & -2.7 & & & & & \\
\hline \multirow[t]{2}{*}{$\begin{array}{l}\text { Fietze } 2007 \text { [11] } \\
17337881\end{array}$} & $\begin{array}{l}42(26) \\
{[\geq 10]}\end{array}$ & nd & $\begin{array}{l}1.5 \text { months } \\
(\mathrm{PL})\end{array}$ & APAP & 20 & nd & nd & nd & nd & NS & 0 & $C$ \\
\hline & & & & CPAP & 21 & nd & nd & & & & & \\
\hline \multirow[t]{2}{*}{$\begin{array}{l}\text { Galetke } 2008 \text { [12] } \\
17148931\end{array}$} & $\begin{array}{l}33(19) \\
{[>10]}\end{array}$ & $10.3(5.7)$ & $\begin{array}{l}2 \text { months } \\
(\mathrm{XO})\end{array}$ & APAP & 20 & $10.3(5.7)$ & -5.4 & -1.7 & $\begin{array}{l}-3.76 \text { to } \\
0.36\end{array}$ & nd & 0 & $C$ \\
\hline & & & & CPAP & 20 & $10.3(5.7)$ & -3.7 & & & & & \\
\hline \multirow[t]{2}{*}{$\begin{array}{l}\text { Hudgel } 2000 \text { [18] } \\
10947032\end{array}$} & $\begin{array}{l}30(25) \\
{[n d]}\end{array}$ & $16.0(5.0)$ & $\begin{array}{l}3 \text { months } \\
(X O)\end{array}$ & APAP & 39 & $16.0(5.0)$ & -7.0 & 1 & $\begin{array}{l}-0.96 \text { to } \\
2.96\end{array}$ & nd & 35 & $C$ \\
\hline & & & & CPAP & 39 & $16.0(5.0)$ & -8.0 & & & & & \\
\hline \multirow[t]{2}{*}{$\begin{array}{l}\text { Hussain } 2004 \text { [20] } \\
15072173\end{array}$} & $\begin{array}{l}47(36) \\
{[>15]}\end{array}$ & $11.1(6.4)$ & $\begin{array}{l}1 \text { months } \\
(X O)\end{array}$ & APAP & 10 & $11.1(6.4)$ & -3.1 & 1.4 & -2.2 to 5.0 & nd & 0 & $C$ \\
\hline & & & & CPAP & 10 & $11.1(6.4)$ & -4.5 & & & & & \\
\hline \multirow[t]{2}{*}{$\begin{array}{l}\text { Hukins } 2004 \text { [19] } \\
15683142\end{array}$} & $\begin{array}{l}56(\mathrm{nd}) \\
{[\geq 5]}\end{array}$ & 12.5 (nd) & $\begin{array}{l}1 \text { to } 2 \text { months } \\
(\mathrm{XO})\end{array}$ & APAP & 46 & $12.5(\mathrm{nd})$ & -4.5 & -0.2 & nd & NS & 16 & $B$ \\
\hline & & & & CPAP & 46 & 12.5 (nd) & -4.3 & & & & & \\
\hline \multirow[t]{2}{*}{$\begin{array}{l}\text { Marrone } 2004 \text { [21] } \\
15165530\end{array}$} & $\begin{array}{l}68(12) \\
{[30]}\end{array}$ & $16.3(5.0)$ & $\begin{array}{l}1 \text { month } \\
(\mathrm{XO})\end{array}$ & APAP & 22 & $16.3(5.0)$ & -12.4 & -1 & -2.4 to 0.4 & nd & 0 & $C$ \\
\hline & & & & CPAP & 22 & $16.3(5.0)$ & -11.4 & & & & & \\
\hline \multirow[t]{2}{*}{$\begin{array}{l}\text { Massie } 2003 \text { [22] } \\
12406840\end{array}$} & $\begin{array}{l}\text { nd } \\
{[\geq 15]}\end{array}$ & nd & $\begin{array}{l}1.5 \text { months } \\
(X O)\end{array}$ & APAP & 44 & nd & nd & -1 & $\begin{array}{l}-2.06 \text { to } \\
0.06^{\mathrm{b}}\end{array}$ & 0.065 & 4 & B \\
\hline & & & & CPAP & 44 & nd & nd & & & & & \\
\hline \multirow[t]{4}{*}{$\begin{array}{l}\text { Meurice } 2007 \text { [13] } \\
17638595\end{array}$} & $\begin{array}{l}55(10) \\
{[n d]}\end{array}$ & $11.8(4.9)$ & $\begin{array}{l}3 \text { months } \\
(\mathrm{PL})\end{array}$ & APAP (AutoSet) & 15 & $12.9(4.3)$ & -9.1 & -3.3 & $\begin{array}{l}-6.68 \text { to } \\
0.08\end{array}$ & NS & 15 & B \\
\hline & & & & CPAP & 14 & $10.6(5.2)$ & -5.8 & & & & & \\
\hline & & & $\begin{array}{l}6 \text { months } \\
(P L)\end{array}$ & APAP (AutoSet) & 15 & $12.9(4.3)$ & -7.7 & -3.0 & $\begin{array}{l}-6.44 \text { to } \\
0.44\end{array}$ & NS & 15 & \\
\hline & & & & CPAP & 14 & $10.6(5.2)$ & -4.7 & & & & & \\
\hline $\begin{array}{l}\text { Nolan } 2007 \text { [14] } \\
17326544\end{array}$ & $\begin{array}{l}15(8) \\
{[\geq 5)}\end{array}$ & $12.3(4.0)$ & $\begin{array}{l}2 \text { months } \\
(\mathrm{XO})\end{array}$ & APAP & 29 & $12.3(4.0)$ & -3.7 & 0.9 & $\begin{array}{l}-0.99 \text { to } \\
2.79^{\mathrm{b}}\end{array}$ & 0.35 & 15 & B \\
\hline
\end{tabular}


Table 4 ESS in randomized controlled trials of APAP versus CPAP (Continued)

\begin{tabular}{|c|c|c|c|c|c|c|c|c|c|c|c|c|c|}
\hline & & & & CPAP & 29 & $12.3(4.0)$ & -4.6 & & & & & & \\
\hline \multirow[t]{2}{*}{$\begin{array}{l}\text { Noseda } 2004 \text { [23] } \\
15249439\end{array}$} & nd [> 20] & $10.7(2.4)$ & $\begin{array}{l}2 \text { months } \\
(X O)\end{array}$ & APAP & 24 & $10.7(2.4)$ & nd & -1 & $\begin{array}{l}-1.76 \text { to } \\
-0.24^{b}\end{array}$ & $\begin{array}{l}< \\
0.01\end{array}$ & 11 & B & 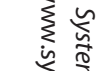 \\
\hline & & & & CPAP & 24 & $10.7(2.4)$ & nd & & & & & & \\
\hline \multirow[t]{2}{*}{$\begin{array}{l}\text { Nussbaumer } 2006 \text { [15] } \\
16537862\end{array}$} & $\begin{array}{l}41(20) \\
{[>10]}\end{array}$ & $12.7(3.29)$ & $\begin{array}{l}1 \text { month } \\
(X O)\end{array}$ & APAP & 30 & $\begin{array}{l}12.7 \\
(0.6)\end{array}$ & -6.6 & 0 & -1.6 to 1.1 & nd & 12 & B & 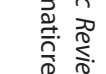 \\
\hline & & & & CPAP & 30 & $\begin{array}{l}12.7 \\
(0.6)\end{array}$ & -6.6 & & & & & & \\
\hline \multirow[t]{2}{*}{$\begin{array}{l}\text { Patruno } 2007 \text { [16] } \\
17494789\end{array}$} & $\begin{array}{l}46(14) \\
{[>20]}\end{array}$ & $15(2.7)$ & $\begin{array}{l}3 \text { months } \\
(\mathrm{PL})\end{array}$ & APAP & 15 & 15.8 (3.5) & nd & nd & nd & NS & 23 & C & \\
\hline & & & & CPAP & 16 & $14.1(1.7)$ & nd & & & & & & \\
\hline \multirow[t]{2}{*}{$\begin{array}{l}\text { Planes } 2003 \text { [29] } \\
12683473\end{array}$} & $\begin{array}{l}59(17) \\
{[\geq 30]}\end{array}$ & 15.1 (3.9) & $\begin{array}{l}2 \text { months } \\
(\mathrm{PL})\end{array}$ & APAP & 16 & $15.5(4.7)$ & -8.0 & -0.9 & $\begin{array}{l}-3.72 \text { to } \\
1.92\end{array}$ & nd & 14 & & 승 \\
\hline & & & & CPAP & 14 & 14.7 (3.9) & -7.1 & & & & & & $\vec{\sigma}$ \\
\hline \multirow[t]{2}{*}{$\begin{array}{l}\text { Randerath } 2001 \text { [26] } \\
11254519\end{array}$} & $\begin{array}{l}35(26) \\
{[\geq 10]}\end{array}$ & $11.1(5.1)$ & $\begin{array}{l}1.5 \text { months } \\
(X O)\end{array}$ & APAP & 52 & $11.1(5.1)$ & -3.3 & -1 & $\begin{array}{l}-2.26 \text { to } \\
0.26\end{array}$ & nd & 12 & B & 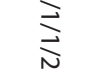 \\
\hline & & & & CPAP & 52 & $11.1(5.1)$ & -2.3 & & & & & & \\
\hline \multirow[t]{2}{*}{$\begin{array}{l}\text { Resta } 2004 \text { [24] } \\
15679008\end{array}$} & $\begin{array}{l}47(11) \\
{[>30]}\end{array}$ & 13.9 (3.2) & $\begin{array}{l}1 \text { month } \\
(\mathrm{PL})\end{array}$ & APAP & 10 & $15.7(5.1)$ & -10.5 & -2.6 & $\begin{array}{l}-5.84 \text { to } \\
0.64\end{array}$ & nd & 0 & C & \\
\hline & & & & CPAP & 10 & $\begin{array}{l}12.0 \\
(3.2)\end{array}$ & -7.9 & & & & & & \\
\hline \multirow{3}{*}{$\begin{array}{l}\text { Senn } 2003 \text { [25] } \\
\text { Switzerland } \\
14525804\end{array}$} & $\begin{array}{l}46(23) \\
{[>10]}\end{array}$ & $14.2(3.77)$ & $\begin{array}{l}1 \text { month } \\
(X O)\end{array}$ & $\begin{array}{l}\text { APAP (Autoset } \\
\text { T) }\end{array}$ & 29 & $14.2(3.77)$ & -5.2 & 0.8 & $\begin{array}{l}-0.49 \text { to } \\
2.09\end{array}$ & nd & 7 & B & \\
\hline & & & & $\begin{array}{l}\text { APAP } \\
\text { (AutoAdjust) }\end{array}$ & 29 & $14.2(3.77)$ & -6.2 & -0.2 & $\begin{array}{l}-1.68 \text { to } \\
1.28\end{array}$ & nd & & & \\
\hline & & & & CPAP & 29 & $14.2(3.77)$ & -6.0 & & & & & & \\
\hline \multirow[t]{4}{*}{$\begin{array}{l}\text { Sériès } 1997 \text { [27] } \\
9341056\end{array}$} & $\begin{array}{l}44(20) \\
{[n d]}\end{array}$ & $15.5(4.5)$ & $\begin{array}{l}0.75 \text { months } \\
\text { (PL) }\end{array}$ & APAP $^{C}$ & 12 & $\begin{array}{l}17.0 \\
(4.1)\end{array}$ & -9.1 & -0.8 & $\begin{array}{l}-4.28 \text { to } \\
2.69\end{array}$ & nd & 0 & C & \\
\hline & & & & CPAP & 12 & $16.1(4.5)$ & -8.3 & & & & & & \\
\hline & & & & APAPd $^{d}$ & 12 & $\begin{array}{l}13.5 \\
(4.7)\end{array}$ & -6.5 & 1.8 & $\begin{array}{l}-1.78 \text { to } \\
5.38\end{array}$ & nd & & & \\
\hline & & & & CPAP & 12 & $\begin{array}{l}16.1 \\
(4.5)\end{array}$ & -8.3 & & & & & & \\
\hline \multirow[t]{4}{*}{$\begin{array}{l}\text { To } 2008[17] \\
18197915\end{array}$} & $\begin{array}{l}54(\mathrm{nd}) \\
{[>30]}\end{array}$ & 13.4 (nd) & $\begin{array}{l}1 \text { month } \\
(\mathrm{XO})\end{array}$ & APAP & 41 & $13.4(5.76)$ & -4.9 & 0.3 & $\begin{array}{l}-1.46 \text { to } \\
2.06\end{array}$ & nd & 5 & B & \\
\hline & & & & CPAP & 41 & $13.4(5.76)$ & -5.2 & & & & & & \\
\hline & & & $\begin{array}{l}2 \text { months } \\
(X O)\end{array}$ & APAP & 41 & $13.4(5.76)$ & -4.9 & 0 & $\begin{array}{l}-1.76 \text { to } \\
1.76\end{array}$ & nd & & & \\
\hline & & & & CPAP & 41 & $13.4(5.76)$ & -4.9 & & & & & & ڤึ \\
\hline \multirow[t]{2}{*}{$\begin{array}{l}\text { Vennelle } 2010 \text { [30] } \\
20175411\end{array}$} & $33(18)[\geq 15]$ & $14(3)$ & 6 weeks (XO) & APAP & 181 & $14(3)$ & -4.5 & -0.5 & $\begin{array}{l}-0.95 \text { to } \\
-0.05\end{array}$ & 0.031 & 9.5 & A & 5 \\
\hline & & & & CPAP & 181 & $14(3)$ & -4 & & & & & & \\
\hline
\end{tabular}


Table 4 ESS in randomized controlled trials of APAP versus CPAP (Continued)

\begin{tabular}{|c|c|c|c|c|c|c|c|c|c|c|}
\hline \multirow[t]{6}{*}{ West 2006 [33] } & $\begin{array}{l}33^{e}(\mathrm{nd}) \\
{[>10]}\end{array}$ & $16.5^{f}(\mathrm{nd})$ & $\begin{array}{l}1 \text { month } \\
(\mathrm{PL})\end{array}$ & APAP & 29 & $\begin{array}{l}16 \\
\text { (median) }\end{array}$ & 7 (final) & 0.9 & 6.1 & A \\
\hline & & & & CPAP (auto) & 31 & $\begin{array}{l}17 \\
\text { (median) }\end{array}$ & 7 (final) & & & \\
\hline & & & & CPAP (algo) & 32 & $\begin{array}{l}16.5 \\
\text { (median) }\end{array}$ & 6 (final) & & & \\
\hline & & & $\begin{array}{l}6 \text { months } \\
\text { (PL) }\end{array}$ & APAP & 28 & $\begin{array}{l}16 \\
\text { (median) }\end{array}$ & 6 (final) & 0.8 & 9.2 & \\
\hline & & & & CPAP (auto) & 31 & $\begin{array}{l}17 \\
\text { (median) }\end{array}$ & 5 (final) & & & \\
\hline & & & & CPAP (algo) & 27 & $\begin{array}{l}16.5 \\
\text { (median) }\end{array}$ & 5 (final) & & & \\
\hline
\end{tabular}

AHI: apnea-hypopnea index (events/hour); algo: fixed pressure CPAP determined by algorithm; APAP: auto-titrating CPAP; auto: fixed pressure CPAP determined by pressures from 1 week of APAP; CPAP: continuous positive airway pressure; ESS: Epworth Sleepiness Scale; nd: no data; NS: non-significant; PL: parallel design; XO: cross-over design. ${ }^{\mathrm{a} E s t i m a t e d ~ f r o m ~ r e p o r t e d ~ d a t a . ~}{ }^{\mathrm{b}}$ Estimated from reported P value. ${ }^{{ }^{C} E s t i m a t e d}$ effective pressure. ${ }^{\mathrm{d}}$ Measured effective pressure. ${ }^{\mathrm{e}}>4 \% \mathrm{SaO}_{2}$ dip rate per hour in algorithm-determined CPAP arm. ${ }^{\mathrm{f}}$ Median in algorithm-determined CPAP arm. 
Table 5 Arousal index (events/hour) in randomized controlled trials of APAP versus CPAP

\begin{tabular}{|c|c|c|c|c|c|c|c|c|c|c|c|c|}
\hline $\begin{array}{l}\text { Study } \\
\text { PMID }\end{array}$ & $\begin{array}{l}\text { Baseline } \\
\text { AHI (SD) } \\
\text { [eligibility] }\end{array}$ & $\begin{array}{l}\text { Baseline } \\
\text { ESS (SD) }\end{array}$ & $\begin{array}{l}\text { Duration } \\
\text { (design) }\end{array}$ & Interventions & $\begin{array}{l}\text { Number } \\
\text { analyzed }\end{array}$ & $\begin{array}{l}\text { Baseline } \\
\text { (SD) }\end{array}$ & $\begin{array}{l}\text { Change } \\
\text { (final) }\end{array}$ & $\begin{array}{l}\text { Net } \\
\text { difference } \\
\text { or } \\
\text { difference }\end{array}$ & $\begin{array}{l}95 \% \\
\mathrm{Cl}^{\mathrm{a}}\end{array}$ & $P$ & $\begin{array}{l}\text { Dropout } \\
\text { (\%) }\end{array}$ & $\begin{array}{l}\text { Study } \\
\text { quality }\end{array}$ \\
\hline \multirow{2}{*}{$\begin{array}{l}d^{\prime} \text { Ortho } \\
2000[32] \\
11035671\end{array}$} & $\begin{array}{l}58(6) \\
{[\geq 10]}\end{array}$ & $12.7(5.3)$ & $\begin{array}{l}2 \text { months } \\
(X O)\end{array}$ & APAP & 25 & $\begin{array}{l}45.6 \\
(25.8)\end{array}$ & -30.1 & 1.8 & nd & NS & 0 & B \\
\hline & & & & CPAP & 25 & $\begin{array}{l}45.6 \\
(25.8)\end{array}$ & -31.9 & & & & & \\
\hline \multirow{4}{*}{$\begin{array}{l}\text { Damjanovic } \\
2009 \text { [10] } \\
19129293\end{array}$} & $\begin{array}{l}44(25) \\
{[\geq 15]}\end{array}$ & $8.8(5.18)$ & $\begin{array}{l}3 \text { months } \\
(\mathrm{PL})\end{array}$ & APAP & 46 & $\begin{array}{l}30.6 \\
(22.4)\end{array}$ & -18.3 & -0.2 & $\begin{array}{l}-7.92 \text { to } \\
7.52\end{array}$ & nd & 8 & B \\
\hline & & & & CPAP & 46 & $\begin{array}{l}34.5 \\
(21.0)\end{array}$ & -18.1 & & & & & \\
\hline & & & $\begin{array}{l}9 \text { months } \\
(\mathrm{PL})\end{array}$ & APAP & 34 & $\begin{array}{l}30.6 \\
(22.4)\end{array}$ & -17.7 & 3.6 & $\begin{array}{l}-4.09 \text { to } \\
11.29\end{array}$ & nd & 22 & \\
\hline & & & & CPAP & 44 & $\begin{array}{l}34.5 \\
(21.0)\end{array}$ & -21.3 & & & & & \\
\hline \multirow{2}{*}{$\begin{array}{l}\text { Galetke } \\
2008 \text { [12] } \\
17148931\end{array}$} & $\begin{array}{l}33(19) \\
{[>10]}\end{array}$ & $10.3(5.7)$ & $\begin{array}{l}2 \text { months } \\
(X O)\end{array}$ & APAP & 20 & $17.6(9.2)$ & -4.0 & 1.0 & $\begin{array}{l}-2.52 \text { to } \\
4.52\end{array}$ & nd & nd & C \\
\hline & & & & CPAP & 20 & $17.6(9.2)$ & -5.0 & & & & & \\
\hline \multirow{2}{*}{$\begin{array}{l}\text { Hussain } \\
2004[20] \\
15072173\end{array}$} & $\begin{array}{l}47(36) \\
{[>15]}\end{array}$ & $11.1(6.4)$ & $\begin{array}{l}1 \text { month } \\
(\mathrm{XO})\end{array}$ & APAP & 10 & $\begin{array}{l}17.3 \\
(17.7)\end{array}$ & -11.4 & 1.0 & $\begin{array}{l}-2.5 \text { to } \\
4.5\end{array}$ & nd & 0 & C \\
\hline & & & & CPAP & 10 & $\begin{array}{l}17.3 \\
(17.7)\end{array}$ & -12.4 & & & & & \\
\hline \multirow[t]{2}{*}{$\begin{array}{l}\text { Konermann } \\
1998[31] \\
9515848\end{array}$} & $\begin{array}{l}38(14) \\
{[>20\}}\end{array}$ & nd & $\begin{array}{l}3 \text { to } 6 \\
\text { months } \\
\text { (PL) }\end{array}$ & APAP & 25 & $\begin{array}{l}16.9 \\
(10.5)\end{array}$ & -9.5 & -3.3 & $\begin{array}{l}-6.6 \text { to } \\
0^{b}\end{array}$ & $\begin{array}{l}< \\
0.05\end{array}$ & 4 & B \\
\hline & & & & CPAP & 23 & $\begin{array}{l}13.3 \\
(13.3)\end{array}$ & -6.2 & & & & & \\
\hline \multirow{2}{*}{$\begin{array}{l}\text { Nolan } 2007 \\
{[14]} \\
17326544\end{array}$} & $\begin{array}{l}15(8) \\
{[\geq 5)}\end{array}$ & $12.3(4)$ & $\begin{array}{l}2 \text { months } \\
(X O)\end{array}$ & APAP & 29 & $\begin{array}{l}16.0 \\
(14.0)\end{array}$ & -14.0 & -3.0 & $\begin{array}{l}-5.7 \text { to } \\
-0.29^{b}\end{array}$ & 0.03 & 15 & B \\
\hline & & & & CPAP & 29 & $\begin{array}{l}16.0 \\
(14.0)\end{array}$ & -11.0 & & & & & \\
\hline \multirow{2}{*}{$\begin{array}{l}\text { Planes } 2003 \\
{[29]} \\
12683473\end{array}$} & $\begin{array}{l}59(17) \\
{[\geq 30]}\end{array}$ & $15.1(3.9)$ & $\begin{array}{l}2 \text { months } \\
(\mathrm{PL})\end{array}$ & APAP & 16 & $\begin{array}{l}44.4 \\
(19.1)\end{array}$ & & 3.3 & $\begin{array}{l}-7.14 \text { to } \\
12.74\end{array}$ & nd & 14 & C \\
\hline & & & & CPAP & 14 & $\begin{array}{l}48.5 \\
(14.2)\end{array}$ & & & & & & \\
\hline \multirow[t]{2}{*}{$\begin{array}{l}\text { Randerath } \\
2001[26] \\
11254519\end{array}$} & $\begin{array}{l}35(26) \\
{[\geq 10]}\end{array}$ & $11.1(5.1)$ & $\begin{array}{l}1.5 \\
\text { months } \\
(\mathrm{XO})\end{array}$ & APAP & 52 & $34(21.7)$ & -23.1 & -1.7 & $\begin{array}{l}-3.7 \text { to } \\
0.3\end{array}$ & nd & 12 & B \\
\hline & & & & CPAP & 52 & $34(21.7)$ & -21.4 & & & & & \\
\hline \multirow{2}{*}{$\begin{array}{l}\text { Resta } 2004 \\
{[24]} \\
15679008\end{array}$} & $\begin{array}{l}47(11) \\
{[>30]}\end{array}$ & $13.9(3.2)$ & $\begin{array}{l}1 \text { month } \\
(\mathrm{PL})\end{array}$ & APAP & 10 & $\begin{array}{l}43.1 \\
(11.9)\end{array}$ & -35.7 & 0.1 & $\begin{array}{l}-8.29 \text { to } \\
8.49\end{array}$ & nd & 0 & C \\
\hline & & & & CPAP & 10 & $43.1(9.1)$ & -35.8 & & & & & \\
\hline \multirow[t]{4}{*}{$\begin{array}{l}\text { Sériès } 1997 \\
{[27]} \\
9341056\end{array}$} & $\begin{array}{l}44(20) \\
{[n d]}\end{array}$ & $15.5(4.5)$ & $\begin{array}{l}0.75 \\
\text { months } \\
\text { (PL) }\end{array}$ & APAP $^{C}$ & 12 & nd & nd & - & - & NS & 0 & C \\
\hline & & & & CPAP & 12 & nd & nd & & & & & \\
\hline & & & & APAP $^{d}$ & 12 & nd & nd & - & - & NS & & \\
\hline & & & & CPAP & 12 & nd & nd & & & & & \\
\hline
\end{tabular}

AHI: apnea-hypopnea index (events/hour); APAP: auto-titrating CPAP; CPAP: continuous positive airway pressure; ESS: Epworth Sleepiness Scale; nd: no data; NS: non-significant; PL: parallel design; XO: cross-over design. ${ }^{a}$ Estimated from reported data. ${ }^{\text {E}}$ Estimated from reported $P$ value. ${ }^{C}$ Estimated effective pressure. ${ }^{\mathrm{d}}$ Measured effective pressure. 
Table 6 Minimum $\mathrm{O}_{2}$ saturation (\%) in randomized controlled trials of APAP versus CPAP

\begin{tabular}{|c|c|c|c|c|c|c|c|c|c|c|c|c|}
\hline $\begin{array}{l}\text { Study } \\
\text { PMID }\end{array}$ & $\begin{array}{l}\text { Baseline } \\
\text { AHI (SD) } \\
\text { [eligibility] }\end{array}$ & $\begin{array}{l}\text { Baseline } \\
\text { ESS (SD) }\end{array}$ & $\begin{array}{l}\text { Duration } \\
\text { (design) }\end{array}$ & Interventions & $\begin{array}{l}\text { Number } \\
\text { analyzed }\end{array}$ & $\begin{array}{l}\text { Baseline } \\
\text { (SD) }\end{array}$ & $\begin{array}{l}\text { Change } \\
\text { (final) }\end{array}$ & $\begin{array}{l}\text { Net } \\
\text { difference } \\
\text { or } \\
\text { difference }\end{array}$ & $\begin{array}{l}95 \% \\
\mathrm{Cl}^{\mathrm{a}}\end{array}$ & $P$ & $\begin{array}{l}\text { Dropout } \\
\text { (\%) }\end{array}$ & $\begin{array}{l}\text { Study } \\
\text { quality }\end{array}$ \\
\hline \multirow{2}{*}{$\begin{array}{l}\text { d'Ortho } 2000 \\
{[32]} \\
11035671\end{array}$} & $\begin{array}{l}58(6) \\
{[\geq 10]}\end{array}$ & $12.7(5.3)$ & $\begin{array}{l}2 \text { months } \\
(X O)\end{array}$ & APAP & 25 & $\begin{array}{l}66.5 \\
(13.6)\end{array}$ & 85.2 & -1.4 & nd & nd & 0 & B \\
\hline & & & & CPAP & 25 & $\begin{array}{l}66.5 \\
(13.6)\end{array}$ & 86.6 & & & & & \\
\hline \multirow{2}{*}{$\begin{array}{l}\text { Galetke } 2008 \\
{[12]} \\
17148931\end{array}$} & $\begin{array}{l}33(19) \\
{[>10]}\end{array}$ & $10.3(5.7)$ & $\begin{array}{l}2 \text { months } \\
(\mathrm{XO})\end{array}$ & APAP & 20 & $77.8(8.4)$ & 8.7 & -1.8 & $\begin{array}{l}-3.8 \\
\text { to } 0.2\end{array}$ & nd & nd & C \\
\hline & & & & CPAP & 20 & $77.8(8.4)$ & 10.5 & & & & & \\
\hline \multirow[t]{2}{*}{$\begin{array}{l}\text { Hussain } 2004 \\
{[20]} \\
15072173\end{array}$} & $\begin{array}{l}47(36) \\
{[>15]}\end{array}$ & $11.1(6.4)$ & $\begin{array}{l}1 \text { month } \\
(\mathrm{XO})\end{array}$ & APAP & 10 & $\begin{array}{l}67.8 \\
(12.5)\end{array}$ & 14 & -3.9 & $\begin{array}{l}-7.3 \\
\text { to } \\
-0.5\end{array}$ & nd & 0 & C \\
\hline & & & & CPAP & 10 & $\begin{array}{l}67.8 \\
(12.5)\end{array}$ & 17.9 & & & & & \\
\hline \multirow[t]{2}{*}{$\begin{array}{l}\text { Konermann } 1998 \\
{[31]} \\
9515848\end{array}$} & $\begin{array}{l}38(14) \\
{[>20\}}\end{array}$ & nd & $\begin{array}{l}3 \text { to } 6 \\
\text { months } \\
(P L)\end{array}$ & APAP & 25 & $\begin{array}{l}76.5 \\
(12.4)\end{array}$ & 90.3 & 1.1 & nd & NS & 4 & B \\
\hline & & & & CPAP & 23 & $\begin{array}{l}74.5 \\
(10.7)\end{array}$ & 87.2 & & & & & \\
\hline \multirow{2}{*}{$\begin{array}{l}\text { Meurice } 2007 \\
{[13]} \\
17638595\end{array}$} & $\begin{array}{l}55(10) \\
{[n d]}\end{array}$ & $11.8(4.9)$ & $\begin{array}{l}6 \text { months } \\
(\mathrm{PL})\end{array}$ & $\begin{array}{l}\text { APAP } \\
\text { (AutoSet) }\end{array}$ & 15 & $\begin{array}{l}82.1 \\
(12.8)\end{array}$ & 0.2 & -2.9 & $\begin{array}{l}-7.1 \\
\text { to } 7.5\end{array}$ & nd & 15 & B \\
\hline & & & & CPAP & 14 & $\begin{array}{l}82.3 \\
(9.9)\end{array}$ & 0.5 & & & & & \\
\hline \multirow[t]{2}{*}{$\begin{array}{l}\text { Nolan } 2007 \text { [14] } \\
17326544\end{array}$} & $\begin{array}{l}15(8) \\
{[\geq 5)}\end{array}$ & $12.3(4)$ & $\begin{array}{l}2 \text { months } \\
(X O)\end{array}$ & APAP & 29 & 79 (11.5) & 8.5 & 4.8 & $\begin{array}{l}-7.4 \\
\text { to } \\
17.0^{b}\end{array}$ & 0.44 & 15 & B \\
\hline & & & & CPAP & 29 & 79 (11.5) & 3.7 & & & & & \\
\hline \multirow[t]{2}{*}{$\begin{array}{l}\text { Patruno } 2007 \\
\text { [16] } 17494789\end{array}$} & $\begin{array}{l}46(14) \\
{[>20]}\end{array}$ & $15(2.7)$ & $\begin{array}{l}3 \text { months } \\
(\mathrm{PL})\end{array}$ & APAP & 15 & $\begin{array}{l}71.7 \\
(10.6)\end{array}$ & 16.4 & -4.4 & $\begin{array}{l}-11.8 \\
\text { to } 3.0\end{array}$ & nd & 23 & C \\
\hline & & & & CPAP & 16 & $\begin{array}{l}70.0 \\
(11.7)\end{array}$ & 20.8 & & & & & \\
\hline \multirow[t]{2}{*}{$\begin{array}{l}\text { Randerath } 2001 \\
{[26]} \\
11254519\end{array}$} & $\begin{array}{l}35(26) \\
{[\geq 10]}\end{array}$ & $11.1(5.1)$ & $\begin{array}{l}1.5 \\
\text { months } \\
(\mathrm{XO})\end{array}$ & APAP & 52 & $81(8.0)$ & 7.0 & -1.0 & $\begin{array}{l}-2.1 \\
\text { to } 0.1\end{array}$ & nd & 12 & $\mathrm{~B}$ \\
\hline & & & & CPAP & 52 & $81(8.0)$ & 8.0 & & & & & \\
\hline \multirow[t]{2}{*}{$\begin{array}{l}\text { Resta } 2004 \text { [24] } \\
15679008\end{array}$} & $\begin{array}{l}47(11) \\
{[>30]}\end{array}$ & $13.9(3.2)$ & $\begin{array}{l}1 \text { month } \\
(\mathrm{PL})\end{array}$ & APAP & 10 & $\begin{array}{l}72.4 \\
(10.5)\end{array}$ & 15.9 & 0.9 & $\begin{array}{l}-7.4 \\
\text { to } 9.2\end{array}$ & nd & 0 & C \\
\hline & & & & CPAP & 10 & $\begin{array}{l}74.1 \\
(10.8)\end{array}$ & 15.0 & & & & & \\
\hline
\end{tabular}

AHI: apnea-hypopnea index (events/hour); APAP: auto-titrating CPAP; CPAP: continuous positive airway pressure; ESS: Epworth Sleepiness Scale; nd: no data; NS: non-significant; PL: parallel design; XO: cross-over design. ${ }^{\mathrm{a}}$ Estimated from reported data. ${ }^{\mathrm{b}}$ Estimated from reported $P$ value.

analysis, which showed a statistically significant difference of 11 minutes per night favoring APAP (difference $=0.18$ hours; $95 \% \mathrm{CI}, 0.05$ to 0.31 minutes; $P=0.006$ ), without statistical heterogeneity (Figure 2).

To test the a priori hypothesis that the relative effect on compliance may differ based on baseline severity, we performed subgroup meta-analyses stratified by minimum AHI threshold. By meta-regression, the subgroups had no significantly different effects from each other. Results were also similar in parallel and cross-over design studies.

\section{Apnea-hypopnea index}

Sixteen trials provided sufficient data for analysis of residual AHI while using treatment (Figure 3) [10-16, $20,22,24-26,28,29,31,32]$. None of the studies reported a statistically significant difference in AHI (events/hour) between APAP and CPAP. The mean net difference in individual studies ranged from -2.8 to 3.5 events/hour, where negative values favor APAP. Meta-analysis across these studies indicated a non-significant difference between APAP and CPAP of 0.25 events/hour (95\% CI, -0.16 to 0.66 events/hour; $P=0.23)$. No statistically 
Table 7 Sleep efficiency (\%) in randomized controlled trials of APAP versus CPAP

\begin{tabular}{|c|c|c|c|c|c|c|c|c|c|c|c|c|}
\hline $\begin{array}{l}\text { Study } \\
\text { PMID }\end{array}$ & $\begin{array}{l}\text { Baseline } \\
\text { AHI (SD) } \\
\text { [eligibility] }\end{array}$ & $\begin{array}{l}\text { Baseline } \\
\text { ESS (SD) }\end{array}$ & $\begin{array}{l}\text { Duration } \\
\text { (design) }\end{array}$ & Interventions & $\begin{array}{l}\text { Number } \\
\text { analyzed }\end{array}$ & $\begin{array}{l}\text { Baseline } \\
\text { (SD) }\end{array}$ & $\begin{array}{l}\text { Change } \\
\text { (final) }\end{array}$ & $\begin{array}{l}\text { Net } \\
\text { difference } \\
\text { or } \\
\text { difference }\end{array}$ & $\begin{array}{l}95 \% \\
\mathrm{Cl}\end{array}$ & $P$ & $\begin{array}{l}\text { Dropout } \\
\text { (\%) }\end{array}$ & $\begin{array}{l}\text { Study } \\
\text { quality }\end{array}$ \\
\hline \multirow[t]{2}{*}{$\begin{array}{l}\text { Konermann } \\
1998[31] \\
9515848\end{array}$} & $\begin{array}{l}38(14) \\
{[>20\}}\end{array}$ & nd & $\begin{array}{l}3 \text { to } 6 \\
\text { months } \\
(P L)\end{array}$ & APAP & 25 & $94.5(5.4)$ & 2 & 6 & nd & NS & 4 & B \\
\hline & & & & CPAP & 23 & $\begin{array}{l}89.2 \\
(13.7)\end{array}$ & -4 & & & & & \\
\hline \multirow[t]{2}{*}{$\begin{array}{l}\text { Nolan } 2007 \\
{[14]} \\
17326544\end{array}$} & $\begin{array}{l}15(8) \\
{[\geq 5)}\end{array}$ & $12.3(4)$ & $\begin{array}{l}2 \text { months } \\
(\mathrm{PL})\end{array}$ & APAP & 29 & $79(9)$ & 4 & -1 & $\begin{array}{l}-4.3 \\
\text { to } \\
2.3^{\mathrm{a}}\end{array}$ & 0.39 & 15 & B \\
\hline & & & & CPAP & 29 & $79(9)$ & 5 & & & & & \\
\hline \multirow[t]{2}{*}{$\begin{array}{l}\text { Resta } 2004 \\
{[24]} \\
15679008\end{array}$} & $\begin{array}{l}47(11) \\
{[>30]}\end{array}$ & $13.9(3.2)$ & $\begin{array}{l}1 \text { month } \\
(\mathrm{PL})\end{array}$ & APAP & 10 & $\begin{array}{l}86.9 \\
(8)\end{array}$ & -0.5 & -2.5 & nd & nd & 0 & $C$ \\
\hline & & & & CPAP & 10 & $\begin{array}{l}84.2 \\
(4.9)\end{array}$ & 2 & & & & & \\
\hline
\end{tabular}

AHI: apnea-hypopnea index (events/hour); APAP: auto-titrating CPAP; CPAP: continuous positive airway pressure; ESS: Epworth Sleepiness Scale; nd: no data; NS: non-significant; $\mathrm{PL}=$ parallel design. ${ }^{\mathrm{a}}$ Estimated from reported data.

significant heterogeneity was observed across studies, despite a broad range in the severity of OSA at baseline. Meta-regression stratified by different minimum AHI thresholds or by study design revealed no apparent differences across subgroups in the relative effects of APAP and CPAP.

\section{Epworth Sleepiness Scale}

Twenty-two trials reported ESS after treatment (Figure 4) $[10-27,29,30,32,33]$. No statistically significant differences in ESS were observed between APAP and CPAP in 20 trials, while two studies reported a significant decrease in ESS favoring APAP [23,30]. The mean net difference in ESS across all studies ranged from -3.3 to 2.0, where negative values favor less sleepiness with APAP. Eighteen trials provided sufficient data for metaanalysis, which yielded a statistically significant difference between APAP and CPAP of -0.48 (95\% CI, -0.81 to $-0.15 ; P=0.005)$, favoring APAP. Despite the broad range of severity of OSA across studies, there was no statistically significant heterogeneity within the overall meta-analysis. Meta-regression stratified by minimum AHI threshold or by study design revealed no apparent differences across subgroups in relative effects of APAP and CPAP.

\section{Other sleep study measures}

Meta-analysis of nine trials showed a non-significant difference in arousal index of -0.85 events/hour (95\% CI, -2.2 to 0.5 events/hour; $P=0.23$ ), favoring APAP (Figure 5 ) $[10,12,14,20,24,26,29,31,32]$. Meta-analysis of nine trials showed a statistically significant difference in minimum oxygen saturation of $-1.3 \%$ (95\% CI, -2.2 to $-0.4 \% ; P=$ 0.003), favoring CPAP (Figure 6) [12-14,16,20,24,26,31,32].
Neither meta-analysis had statistically significant heterogeneity. Meta-regression revealed no differences across AHI or study design subgroups.

The three trials reporting on sleep efficiency (percentage of time asleep while in bed) after a period of treatment did not find a statistically significant difference between APAP and CPAP for improvement in the percentage of time spent asleep [14,24,31]. Nine trials reporting on percentage of time spent in REM sleep did not find statistically significant differences between groups $[13,14,20,24,26,27,29,31,32]$. Seven of eight trials found no statistically significant difference in slow wave sleep (stages 3 or 4 ) $[13,14,20,24,26,27,31,32]$. The one outlier reported a statistically significant net mean increase of $7.8 \%$ (95\% CI, 1.8 to $13.7 \%$; $P<0.01$ ); patients using APAP spent more time in slow wave sleep [31]. No study reported effect on the multiple sleep latency test.

\section{Quality of life}

Nine trials evaluated quality of life measures $[11,13,15,17,19,22,25,30,33]$. One trial that included the SF-36 ${ }^{\circledR}$ (Short Form Health Survey) found a significant difference in the mental health (net difference of 5 points; $95 \% \mathrm{CI}, 0.2$ to 9.8 points; $P<0.05$ ) and vitality (net difference of 7 points; $95 \% \mathrm{CI}, 0.6$ to 13.4 points; $P$ $<0.05)$ components, favoring those who used APAP [22]. No other significant differences in quality of life measures between APAP and CPAP were reported in this or the other eight trials. One trial reported on the Sleep Apnea Quality of Life Index and found no difference between groups [33]. No study reported any effect on the Functional Outcomes Sleep Questionnaire. Due to the heterogeneity of specific quality of life outcome 
Table 8 Rapid eye movement sleep (\%) in randomized controlled trials of APAP versus CPAP

\begin{tabular}{|c|c|c|c|c|c|c|c|c|c|c|c|c|}
\hline $\begin{array}{l}\text { Study } \\
\text { PMID }\end{array}$ & $\begin{array}{l}\text { Baseline } \\
\text { AHI (SD) } \\
\text { [eligibility] }\end{array}$ & $\begin{array}{l}\text { Baseline } \\
\text { ESS (SD) }\end{array}$ & $\begin{array}{l}\text { Duration } \\
\text { (design) }\end{array}$ & Interventions & $\begin{array}{l}\text { Number } \\
\text { analyzed }\end{array}$ & $\begin{array}{l}\text { Baseline } \\
\text { (SD) }\end{array}$ & $\begin{array}{l}\text { Change } \\
\text { (final) }\end{array}$ & $\begin{array}{l}\text { Net } \\
\text { difference } \\
\text { or } \\
\text { difference }\end{array}$ & $\begin{array}{l}95 \% \\
\mathrm{Cl}^{\mathrm{a}}\end{array}$ & $P$ & $\begin{array}{l}\text { Dropout } \\
\text { (\%) }\end{array}$ & $\begin{array}{l}\text { Study } \\
\text { quality }\end{array}$ \\
\hline \multirow{2}{*}{$\begin{array}{l}\text { d'Ortho } 2000 \\
{[32]} \\
11035671\end{array}$} & $\begin{array}{l}58(6) \\
{[\geq 10]}\end{array}$ & $12.7(5.3)$ & $\begin{array}{l}2 \text { months } \\
(X O)\end{array}$ & APAP & 25 & $\begin{array}{l}16 \\
(5)\end{array}$ & 5 & -1 & nd & nd & 0 & B \\
\hline & & & & CPAP & 25 & $\begin{array}{l}16 \\
(5)\end{array}$ & 6 & & & & & \\
\hline \multirow[t]{2}{*}{$\begin{array}{l}\text { Hussain } 2004 \\
{[20]} \\
15072173\end{array}$} & $\begin{array}{l}47(36) \\
{[>15]}\end{array}$ & $11.1(6.4)$ & $\begin{array}{l}1 \text { month } \\
(\mathrm{XO})\end{array}$ & APAP & 10 & $15(7.0)$ & 4.0 & -1 & $\begin{array}{l}-4.72 \\
\text { to } \\
2.72\end{array}$ & nd & 0 & C \\
\hline & & & & CPAP & 10 & $17.6(5.1)$ & 2.0 & & & & & \\
\hline \multirow[t]{2}{*}{$\begin{array}{l}\text { Konermann } \\
1998[31] \\
9515848\end{array}$} & $\begin{array}{l}38(14) \\
{[>20]}\end{array}$ & nd & $\begin{array}{l}3 \text { to } 6 \\
\text { months } \\
(\mathrm{PL})\end{array}$ & APAP & 25 & $\begin{array}{l}8.2 \\
(8.1)\end{array}$ & 11.9 & 7.1 & nd & NS & 4 & B \\
\hline & & & & CPAP & 23 & $\begin{array}{l}5.4 \\
(6.0)\end{array}$ & 4.8 & & & & & \\
\hline \multirow[t]{2}{*}{$\begin{array}{l}\text { Meurice } 2007 \\
{[13]} \\
17638595\end{array}$} & $\begin{array}{l}55(10) \\
{[n d]}\end{array}$ & $11.8(4.9)$ & $\begin{array}{l}6 \text { months } \\
(\mathrm{PL})\end{array}$ & $\begin{array}{l}\text { APAP } \\
\text { (AutoSet) }\end{array}$ & 15 & $18.9(6.6)$ & -2.4 & -2.9 & $\begin{array}{l}-7.49 \\
\text { to } \\
1.69\end{array}$ & nd & 15 & B \\
\hline & & & & CPAP & 14 & $\begin{array}{l}19.1 \\
(5.9)\end{array}$ & 0.5 & & & & & \\
\hline \multirow[t]{2}{*}{$\begin{array}{l}\text { Nolan } 2007 \text { [14] } \\
17326544\end{array}$} & $\begin{array}{l}15(8) \\
{[\geq 5)}\end{array}$ & $12.3(4)$ & $\begin{array}{l}2 \text { months } \\
(X O)\end{array}$ & APAP & 29 & $17.6(5.1)$ & -0.5 & -2.5 & $\begin{array}{l}-5.11 \\
\text { to } \\
0.11^{b}\end{array}$ & 0.06 & 15 & B \\
\hline & & & & CPAP & 29 & $17.6(5.1)$ & 2.0 & & & & & \\
\hline \multirow[t]{2}{*}{$\begin{array}{l}\text { Planes } 2003 \\
{[29]} \\
12683473\end{array}$} & $\begin{array}{l}59(17) \\
{[\geq 30]}\end{array}$ & $15.1(3.9)$ & $\begin{array}{l}2 \text { months } \\
(\mathrm{PL})\end{array}$ & APAP & 16 & $\begin{array}{l}12.4 \\
(7.0)\end{array}$ & 4.2 & 0.5 & $\begin{array}{l}-5.44 \\
\text { to } \\
6.44\end{array}$ & nd & 14 & C \\
\hline & & & & CPAP & 14 & $13.7(9.3)$ & 3.7 & & & & & \\
\hline \multirow[t]{2}{*}{$\begin{array}{l}\text { Randerath } 2001 \\
{[26]} \\
11254519\end{array}$} & $\begin{array}{l}35(26) \\
{[\geq 10]}\end{array}$ & $11.1(5.1)$ & $\begin{array}{l}1.5 \\
\text { months } \\
(X O)\end{array}$ & APAP & 52 & $11(8)$ & 6.0 & 1 & $\begin{array}{l}-0.63 \\
\text { to } \\
2.63\end{array}$ & nd & 12 & B \\
\hline & & & & CPAP & 52 & $11(8)$ & 5.0 & & & & & \\
\hline \multirow[t]{2}{*}{$\begin{array}{l}\text { Resta } 2004 \text { [24] } \\
15679008\end{array}$} & $\begin{array}{l}47(11) \\
{[>30]}\end{array}$ & $13.9(3.2)$ & $\begin{array}{l}1 \text { month } \\
\text { (PL) }\end{array}$ & APAP & 10 & $\begin{array}{l}15.0 \\
(8.1)\end{array}$ & 6.7 & -2 & $\begin{array}{l}-10.31 \\
\text { to } \\
6.31\end{array}$ & nd & 0 & C \\
\hline & & & & CPAP & 10 & $15.9(4.2)$ & 8.7 & & & & & \\
\hline \multirow[t]{4}{*}{$\begin{array}{l}\text { Sériès } 1997 \text { [27] } \\
9341056\end{array}$} & $\begin{array}{l}44(20) \\
{[n d]}\end{array}$ & $15.5(4.5)$ & $\begin{array}{l}0.75 \\
\text { months } \\
(P L)\end{array}$ & APAPC $^{C}$ & 12 & nd & $n d^{d}$ & - & - & NS & 0 & C \\
\hline & & & & CPAP & 12 & nd & nd & & & & & \\
\hline & & & & APAPe & 12 & nd & $n d^{d}$ & - & - & NS & & \\
\hline & & & & CPAP & 12 & nd & nd & & & & & \\
\hline
\end{tabular}

AHI: apnea-hypopnea index (events/hour); APAP: auto-titrating CPAP; CPAP: continuous positive airway pressure; ESS: Epworth Sleepiness Scale; nd: no data; NS: non-significant; PL: parallel design; XO: cross-over design. ${ }^{\mathrm{a} E s t i m a t e d ~ f r o m ~ r e p o r t e d ~ d a t a . ~}{ }^{\mathrm{b}}$ Estimated from reported $P$ value. ${ }^{\mathrm{C}}$ Estimated effective pressure.

${ }^{\mathrm{d}}$ Directions of changes not reported in the study. ${ }^{\mathrm{e}}$ Measured effective pressure.

reported (13 among these studies, including the components of SF-36), in Table 10 we summarized only whether any measure statistically favored APAP or $\mathrm{CPAP}$ and if so, what the net difference was.

\section{Blood pressure}

Three trials reported changes in blood pressure $[14,16,33]$. Two studies did not find significant differences in blood pressure changes between the APAP and CPAP groups $[14,33]$. In the third study [16], based on reported data, we estimated a non-significant greater reduction in systolic blood pressure (net difference $=6$ $\mathrm{mmHg}$; $95 \% \mathrm{CI},-1$ to $13 \mathrm{mmHg} P=0.09$ ) and a significant greater reduction in diastolic blood pressure (net difference $=8 \mathrm{mmHg} ; 95 \% \mathrm{CI}, 4$ to $11 \mathrm{mmHg} ; P<$ 0.001) with CPAP compared to APAP.

\section{Adverse effects}

No trials reported any unexpected adverse effects with positive airway treatments. Seven trials reported 
Table 9 Stage 3 or 4 sleep (\%) in randomized controlled trials of APAP versus CPAP

\begin{tabular}{|c|c|c|c|c|c|c|c|c|c|c|c|c|}
\hline $\begin{array}{l}\text { Study } \\
\text { PMID }\end{array}$ & $\begin{array}{l}\text { Baseline } \\
\text { AHI (SD) } \\
\text { [eligibility] }\end{array}$ & $\begin{array}{l}\text { Baseline } \\
\text { ESS (SD) }\end{array}$ & $\begin{array}{l}\text { Duration } \\
\text { (design) }\end{array}$ & Interventions & $\begin{array}{l}\text { Number } \\
\text { analyzed }\end{array}$ & $\begin{array}{l}\text { Baseline } \\
\text { (SD) }\end{array}$ & $\begin{array}{l}\text { Change } \\
\text { (final) }\end{array}$ & $\begin{array}{l}\text { Net } \\
\text { difference } \\
\text { or } \\
\text { difference }\end{array}$ & $\begin{array}{l}95 \% \\
\mathrm{Cl}^{\mathrm{a}}\end{array}$ & $P$ & $\begin{array}{l}\text { Dropout } \\
\text { (\%) }\end{array}$ & $\begin{array}{l}\text { Study } \\
\text { quality }\end{array}$ \\
\hline \multirow{2}{*}{$\begin{array}{l}\text { d'Ortho } \\
2000 \text { [32] } \\
11035671\end{array}$} & $\begin{array}{l}58(6) \\
{[\geq 10]}\end{array}$ & $12.7(5.3)$ & $\begin{array}{l}2 \text { months } \\
(X O)\end{array}$ & APAP & 25 & $\begin{array}{l}44 \min \\
(36)\end{array}$ & 43 & $-9 \min$ & nd & NS & 0 & B \\
\hline & & & & CPAP & 25 & $\begin{array}{l}44 \min \\
(36)\end{array}$ & 52 & & & & & \\
\hline \multirow{2}{*}{$\begin{array}{l}\text { Hussain } \\
2004[20] \\
15072173\end{array}$} & $\begin{array}{l}47(36) \\
{[>15]}\end{array}$ & $11.1(6.4)$ & $\begin{array}{l}1 \text { month } \\
(\mathrm{XO})\end{array}$ & APAP & 10 & $14(25)$ & -4.0 & -8 & $\begin{array}{l}-18.32 \\
\text { to } 2.32\end{array}$ & nd & 0 & C \\
\hline & & & & CPAP & 10 & $14(25)$ & 4.0 & & & & & \\
\hline \multirow[t]{2}{*}{$\begin{array}{l}\text { Konermann } \\
1998[31] \\
9515848\end{array}$} & $\begin{array}{l}38(14) \\
{[>20]}\end{array}$ & nd & $\begin{array}{l}3 \text { to } 6 \\
\text { months } \\
(P L)\end{array}$ & APAP & 25 & $\begin{array}{l}13.2 \\
(12.2)\end{array}$ & 14 & 7.8 & $\begin{array}{l}1.8 \text { to } \\
13.7^{b}\end{array}$ & $\begin{array}{l}< \\
0.01\end{array}$ & 4 & B \\
\hline & & & & CPAP & 23 & $\begin{array}{l}11.4 \\
(10.4)\end{array}$ & 6.2 & & & & & \\
\hline \multirow{2}{*}{$\begin{array}{l}\text { Meurice } \\
2007 \text { [13] } \\
17638595\end{array}$} & $\begin{array}{l}55(10) \\
{[n d]}\end{array}$ & $11.8(4.9)$ & $\begin{array}{l}6 \text { months } \\
(\mathrm{PL})\end{array}$ & $\begin{array}{l}\text { APAP } \\
\text { (AutoSet) }\end{array}$ & 15 & $\begin{array}{l}25.5 \\
(14.7)\end{array}$ & -4.4 & -5.2 & $\begin{array}{l}-13.51 \\
\text { to } 3.11\end{array}$ & nd & 15 & B \\
\hline & & & & CPAP & 14 & $\begin{array}{l}17.1 \\
(7)\end{array}$ & 0.8 & & & & & \\
\hline \multirow{2}{*}{$\begin{array}{l}\text { Nolan } 2007 \\
{[14]} \\
17326544\end{array}$} & $\begin{array}{l}15(8) \\
{[\geq 5)}\end{array}$ & $12.3(4)$ & $\begin{array}{l}2 \text { months } \\
(\mathrm{XO})\end{array}$ & APAP & 29 & $13.7(7.8)$ & 1.3 & 0.3 & $\begin{array}{l}-3.29 \text { to } \\
3.89^{\mathrm{b}}\end{array}$ & 0.87 & 15 & B \\
\hline & & & & CPAP & 29 & $13.7(7.8)$ & 1.0 & & & & & \\
\hline \multirow[t]{2}{*}{$\begin{array}{l}\text { Randerath } \\
2001 \text { [26] } \\
11254519\end{array}$} & $\begin{array}{l}35(26) \\
{[\geq 10]}\end{array}$ & $11.1(5.1)$ & $\begin{array}{l}1.5 \\
\text { months } \\
(X O)\end{array}$ & APAP & 52 & $14(11)$ & 0 & -1 & $\begin{array}{l}-3.45 \text { to } \\
1.45\end{array}$ & NS & 12 & B \\
\hline & & & & CPAP & 52 & $14(11)$ & 1.0 & & & & & \\
\hline \multirow{2}{*}{$\begin{array}{l}\text { Resta } 2004 \\
{[24]} \\
15679008\end{array}$} & $\begin{array}{l}47(11) \\
{[>30]}\end{array}$ & $13.9(3.2)$ & $\begin{array}{l}1 \text { month } \\
\text { (PL) }\end{array}$ & APAP & 10 & $\begin{array}{l}19.8 \\
(10.9)\end{array}$ & 14 & 7.3 & $\begin{array}{l}-2.35 \text { to } \\
16.95\end{array}$ & nd & 0 & C \\
\hline & & & & CPAP & 10 & $\begin{array}{l}22.8 \\
(12.5)\end{array}$ & 6.7 & & & & & \\
\hline \multirow[t]{4}{*}{$\begin{array}{l}\text { Sériès } 1997 \\
\text { [27] } \\
9341056\end{array}$} & $\begin{array}{l}44(20) \\
{[n d]}\end{array}$ & $15.5(4.5)$ & $\begin{array}{l}0.75 \\
\text { months } \\
(\mathrm{PL})\end{array}$ & APAPC & 12 & nd & $n d^{d}$ & - & - & NS & 0 & C \\
\hline & & & & CPAP & 12 & nd & nd & & & & & \\
\hline & & & & APAPe & 12 & nd & $n d^{d}$ & - & - & NS & & \\
\hline & & & & CPAP & 12 & nd & nd & & & & & \\
\hline
\end{tabular}

AHI: apnea-hypopnea index (events/hour); APAP: auto-titrating CPAP; CPAP; continuous positive airway pressure; ESS: Epworth Sleepiness Scale; nd: no data; NS: non-significant; PL: parallel design; XO: cross-over design. ${ }^{\mathrm{a} E s t i m a t e d ~ f r o m ~ r e p o r t e d ~ d a t a . ~}{ }^{\mathrm{b}}$ Estimated from reported $P$ value. ${ }^{\mathrm{C}}$ Estimated effective pressure. ${ }^{\mathrm{d}}$ Directions of changes not reported in the study. ${ }^{\mathrm{e}}$ Measured effective pressure.

quantitative comparisons of treatment-related side effects between groups $[12,14,15,17,19,25,26]$. The side effects were dry mouth, air leakage, skin or nasal-oral irritation. No differences in side effects were reported in five trials $[12,14,15,25,26]$. Two trials reported that the use of APAP was associated with significantly fewer treatment-related side effects $[17,19]$.

\section{Discussion}

Despite the lack of evidence on objective clinical outcomes, given the largely similar magnitude of effects between APAP and CPAP on sleep measures and wakefulness assessment and the relatively small increase (even though statistically significant) in compliance of about 11 minutes with APAP, we concluded that the overall strength of evidence is moderate that APAP and CPAP result in largely similar treatment effects for patients with OSA (Table 11).

The aim of this study was to systematically compare the treatment effects of APAP versus fixed CPAP. Twenty-four trials that included over 1,000 patients provided evidence that APAP reduces sleepiness as measured by ESS by approximately 0.5 points more than fixed CPAP. For compliance, there was a statistically 
Table 10 Quality of life and functional outcomes in randomized controlled trials of APAP versus CPAP

\begin{tabular}{|c|c|c|c|c|c|c|c|c|c|c|c|c|c|c|}
\hline \multirow{3}{*}{$\begin{array}{l}\text { Study } \\
\text { PMID }\end{array}$} & \multirow{3}{*}{$\begin{array}{c}\text { Baseline AHI } \\
\text { (SD) } \\
\text { [eligibility] }\end{array}$} & \multirow{3}{*}{$\begin{array}{l}\text { Baseline ESS } \\
\text { (SD) }\end{array}$} & \multirow[t]{3}{*}{ Interventions } & \multirow{3}{*}{$\begin{array}{l}\text { Number } \\
\text { analyzed }\end{array}$} & \multirow{3}{*}{$\begin{array}{l}\text { Duration } \\
\text { (design) }\end{array}$} & \multirow[t]{3}{*}{ Outcome } & \multirow[t]{3}{*}{ Favors } & \multicolumn{5}{|c|}{ If significant difference } & \multirow{3}{*}{$\begin{array}{l}\text { Dropout } \\
\text { (\%) }\end{array}$} & \multirow{3}{*}{$\begin{array}{l}\text { Study } \\
\text { quality }\end{array}$} \\
\hline & & & & & & & & \multirow{2}{*}{$\begin{array}{c}\text { Net } \\
\text { difference }\end{array}$} & \multirow[t]{2}{*}{$95 \% \mathrm{Cl}$} & \multicolumn{2}{|c|}{ Test range } & \multirow[t]{2}{*}{$P$} & & \\
\hline & & & & & & & & & & "Worst" & "Best" & & & \\
\hline \multirow[t]{2}{*}{$\begin{array}{l}\text { Fietze } 2007 \text { [11] } \\
17337881\end{array}$} & $\begin{array}{c}42(26) \\
{[\geq 10]}\end{array}$ & nd & APAP & 20 & $\begin{array}{l}1.5 \text { months } \\
(\mathrm{XO})\end{array}$ & $\begin{array}{l}\text { SF-36 } \\
\text { all }\end{array}$ & 0 & & & & & & 0 & $C$ \\
\hline & & & CPAP & 21 & & & & & & & & & & \\
\hline \multirow[t]{3}{*}{$\begin{array}{l}\text { Hukins } 2004 \text { [19] } \\
15683142\end{array}$} & $\begin{array}{c}56 \text { (nd) } \\
{[\geq 5]}\end{array}$ & 12.5 (nd) & APAP & 46 & $\begin{array}{c}2 \text { months } \\
(\mathrm{XO})\end{array}$ & SF-36-M & 0 & & & & & & 16 & B \\
\hline & & & CPAP & 46 & & & & & & & & & & \\
\hline & & & & & & SF-36-P & 0 & & & & & & & \\
\hline \multirow[t]{3}{*}{$\begin{array}{l}\text { Massie } 2003 \text { [22] } \\
12406840\end{array}$} & $\begin{array}{l}\text { nd } \\
{[\geq 15]}\end{array}$ & nd & APAP & 44 & $\begin{array}{l}1.5 \text { months } \\
(\mathrm{XO})\end{array}$ & SF-36-MH & APAP & 5 & $\begin{array}{l}0.16 \text { to } \\
9.8^{a}\end{array}$ & 0 & 100 & $\begin{array}{c}< \\
0.05\end{array}$ & 4 & B \\
\hline & & & CPAP & 44 & & SF-36 - vitality & APAP & 7 & $\begin{array}{l}0.6 \text { to } \\
13.4^{\mathrm{a}}\end{array}$ & 0 & 100 & $\begin{array}{c}< \\
0.05\end{array}$ & & \\
\hline & & & & & & $\begin{array}{l}\text { SF-36 - } \\
\text { remainder }\end{array}$ & 0 & & & & & & & \\
\hline \multirow[t]{4}{*}{$\begin{array}{l}\text { Meurice } 2007 \text { [13] } \\
17638595\end{array}$} & $\begin{array}{l}55(10) \\
{[n d]}\end{array}$ & $11.8(4.9)$ & $\begin{array}{c}\text { APAP } \\
\text { (AutoSet) }\end{array}$ & 15 & $\begin{array}{c}3 \text { months } \\
(\mathrm{PL})\end{array}$ & SF-36-M & 0 & & & & & & 15 & B \\
\hline & & & CPAP & 14 & & SF-36-P & 0 & & & & & & & \\
\hline & & & & & $\begin{array}{c}6 \text { months } \\
(\mathrm{PL})\end{array}$ & SF-36-M & 0 & & & & & & 15 & \\
\hline & & & & & & SF-36-P & 0 & & & & & & & \\
\hline \multirow{2}{*}{$\begin{array}{l}\text { Nussbaumer } 2006 \\
{[15]} \\
16537862\end{array}$} & $\begin{array}{l}41(20) \\
{[>10]}\end{array}$ & $12.7(3.3)$ & APAP & 30 & $\begin{array}{l}1 \text { month } \\
(\mathrm{XO})\end{array}$ & $\begin{array}{l}\text { SF-36 } \\
\text { all }\end{array}$ & 0 & & & & & & 12 & $\mathrm{~B}$ \\
\hline & & & CPAP & 30 & & & & & & & & & & \\
\hline \multirow[t]{3}{*}{$\begin{array}{l}\text { Senn } 2003 \text { [25] } \\
14525804\end{array}$} & $\begin{array}{l}46(23) \\
{[>10]}\end{array}$ & $14.2(3.8)$ & $\begin{array}{c}\text { APAP } \\
\text { (Autoset T) }\end{array}$ & 29 & 1 month & $\begin{array}{l}\text { SF-36 } \\
\text { all }\end{array}$ & 0 & & & & & & 7 & B \\
\hline & & & $\begin{array}{c}\text { APAP } \\
\text { (AutoAdjust) }\end{array}$ & 29 & & $\begin{array}{l}\text { Vigilance } \\
\text { (OSLER) }\end{array}$ & 0 & & & & & & & \\
\hline & & & CPAP & 29 & & & & & & & & & & \\
\hline \multirow[t]{2}{*}{$\begin{array}{l}\text { To } 2008 \text { [17] } \\
18197915\end{array}$} & $\begin{array}{c}54.3(\mathrm{nd}) \\
{[>30]}\end{array}$ & 13.4 (nd) & APAP & 41 & $\begin{array}{l}1 \text { month } \\
(\mathrm{XO})\end{array}$ & SAQLI & 0 & & & & & & 5 & $\mathrm{~B}$ \\
\hline & & & CPAP & 41 & $\begin{array}{c}2 \text { months } \\
(X O)\end{array}$ & SAQLI & 0 & & & & & & & \\
\hline \multirow{3}{*}{$\begin{array}{l}\text { Vennelle } 2010 \text { [30] } \\
20175411\end{array}$} & $33(18)[\geq 15]$ & $14(3)$ & APAP & 181 & 6 weeks $(X O)$ & SF-36-M & 0 & & & & & & 9.5 & A \\
\hline & & & CPAP & 181 & & SF-36-P & 0 & & & & & & & \\
\hline & & & & & & $\begin{array}{l}\text { Vigilance } \\
\text { (OSLER) }\end{array}$ & 0 & & & & & & & \\
\hline
\end{tabular}


Table 10 Quality of life and functional outcomes in randomized controlled trials of APAP versus CPAP (Continued)

\begin{tabular}{|c|c|c|c|c|c|c|c|c|}
\hline & & & & & & $\begin{array}{c}\text { Vigilance } \\
\text { (Psychomotor) }\end{array}$ & 0 & \\
\hline \multirow[t]{4}{*}{$\begin{array}{l}\text { West } 2006 \text { [33] } \\
16254055\end{array}$} & $\begin{array}{c}33^{b}(\mathrm{nd}) \\
{[>10]}\end{array}$ & $16.5^{c}(\mathrm{nd})$ & APAP & 28 & 6 months (PL) & SF-36-P & 0 & 9.2 \\
\hline & & & CPAP (auto) & 31 & & SF-36-M & 0 & \\
\hline & & & CPAP (algo) & 27 & & $\begin{array}{l}\text { SF-36-energy, } \\
\text { vitality }\end{array}$ & 0 & \\
\hline & & & & & & $\begin{array}{l}\text { Vigilance } \\
\text { (OSLER) }\end{array}$ & 0 & \\
\hline
\end{tabular}

AHI: apnea-hypopnea index (events/hour); algo: fixed pressure CPAP determined by algorithm; APAP: auto-titrating CPAP; auto: fixed pressure CPAP determined by pressures from 1 week of APAP; CPAP: continuous positive airway pressure; ESS: Epworth Sleepiness Scale; nd: no data; NS: non-significant; PL: parallel design; OSLER: The Oxford Sleep Resistance Test; SAQLI: Sleep Apnea Quality of Life Index; XO: cross-over design.

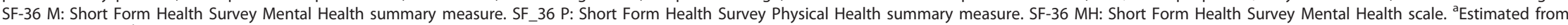
reported P value. ${ }^{b}>4 \% \mathrm{SaO} 2$ dip rate per hour in algorithm-determined CPAP arm. ${ }^{c}$ Median in algorithm-determined CPAP arm. 


\begin{tabular}{|c|c|c|c|c|c|c|c|c|}
\hline $\begin{array}{l}\text { Minimum AHI } \\
\text { Study (Year) }\end{array}$ & & Difference $(95 \% \mathrm{Cl})$ & Months & $\underset{\text { APAP }}{N}$ & $\stackrel{N}{\text { Fixed }}$ & $\begin{array}{l}\text { Base } \\
\text { AHI }\end{array}$ & $\begin{array}{l}\text { Compliance } \\
\text { Fixed }\end{array}$ & Quality \\
\hline $\begin{array}{l}\text { AHI } \geq 5 \text { events } / \mathrm{hr} \\
\text { Nolan }(2007)\end{array}$ & $\rightarrow 1$ & $0.00(-0.59,0.59)$ & 2 & 29 & 29 & 15 & 4.9 & B \\
\hline 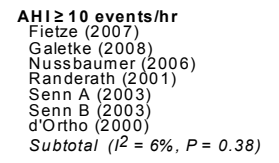 & \begin{tabular}{r|l} 
\\
\multirow{2}{*}{1} \\
\multirow{2}{*}{}
\end{tabular} & $\begin{array}{l}0.80(-0.02,1.62) \\
-0.01(-0.82,0.80) \\
0.30(-0.29,0.89) \\
0.00(-0.44,0.44) \\
-0.10(-0.69,0.49) \\
-0.10(-0.69,0.49) \\
-0.60(-1.52,0.32) \\
0.04(-0.20,0.29)\end{array}$ & $\begin{array}{l}1.5 \\
2 \\
1 \\
1.5 \\
1 \\
1 \\
2\end{array}$ & $\begin{array}{l}20 \\
20 \\
30 \\
52 \\
29 \\
29 \\
25\end{array}$ & $\begin{array}{l}21 \\
20 \\
30 \\
52 \\
29 \\
29 \\
25\end{array}$ & $\begin{array}{l}42 \\
33 \\
41 \\
35 \\
46 \\
46 \\
58\end{array}$ & $\begin{array}{l}4.2 \\
6.4 \\
4.8 \\
5.3 \\
5.6 \\
5.6 \\
4.7\end{array}$ & $\begin{array}{l}C \\
C \\
C \\
B \\
B \\
B \\
B \\
B\end{array}$ \\
\hline 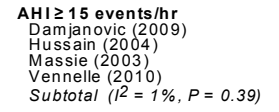 & 2 & $\begin{array}{l}0.10(-0.88,1.08) \\
-0.60(-0.84,2.04) \\
0.58(0.17,0.99) \\
0.20(0.00,0.40) \\
0.28(0.10,0.46)\end{array}$ & $\begin{array}{l}9 \\
1 \\
1.5 \\
1.5\end{array}$ & $\begin{array}{l}34 \\
10 \\
44 \\
181\end{array}$ & $\begin{array}{l}44 \\
10 \\
44 \\
181\end{array}$ & $\begin{array}{l}44 \\
47 \\
33\end{array}$ & $\begin{array}{l}5.1 \\
3.7 \\
4.5 \\
4.2\end{array}$ & $\begin{array}{l}B \\
C \\
B \\
A\end{array}$ \\
\hline $\begin{array}{l}\text { AHI } 220 \text { events/hr } \\
\text { Konermann }(1998) \\
\text { Noseda } 2004) \\
\text { Patruno }(20007) \\
\text { Teschler }(2000) \\
\text { Subtotal }(12=0 \%, P=0.77)\end{array}$ & 2 & $\begin{array}{l}0.30(-0.59,1.19) \\
-0.20(-0.89,0.49) \\
0.20(-0.25,0.65) \\
0.20(-0.70,1.10) \\
0.13(-0.20,0.45)\end{array}$ & $\begin{array}{l}3-6 \\
2 \\
3 \\
2\end{array}$ & $\begin{array}{l}25 \\
24 \\
15 \\
10\end{array}$ & $\begin{array}{l}23 \\
24 \\
16 \\
10\end{array}$ & $\begin{array}{l}38 \\
51 \\
46 \\
53\end{array}$ & $\begin{array}{l}5.7 \\
5.5 \\
6.1 \\
6.1\end{array}$ & $\begin{array}{l}B \\
\text { B } \\
\text { C } \\
C\end{array}$ \\
\hline 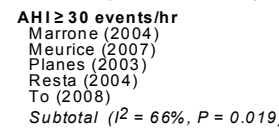 & $\longrightarrow \longrightarrow$ & $\begin{array}{l}0.50(-0.26,1.26) \\
-0.40-1.28,0.48) \\
-0.80(-1.62,0.02) \\
-0.10(-1.1,2,0.92) \\
0.70(0.17,1.23) \\
0.03(-0.57,0.63)\end{array}$ & $\begin{array}{l}1 \\
6 \\
2 \\
1 \\
2\end{array}$ & $\begin{array}{l}22 \\
15 \\
16 \\
10 \\
41\end{array}$ & $\begin{array}{l}22 \\
14 \\
14 \\
10 \\
41\end{array}$ & $\begin{array}{l}68 \\
55 \\
59 \\
47 \\
54\end{array}$ & $\begin{array}{l}4.4 \\
6.1 \\
5.3 \\
5.3 \\
3.7\end{array}$ & $\begin{array}{l}\text { C } \\
\text { B } \\
C \\
C \\
B\end{array}$ \\
\hline $\begin{array}{l}\text { No data } \\
\text { Hudgel }(2000) \\
\text { Hukins }(2004) \\
\text { Subtotal }\left(I^{2}=21 \%, P=0.26\right)\end{array}$ & $\stackrel{1}{\longrightarrow}$ & $\begin{array}{l}0.50(0.02,0.98) \\
0.19(-0.06,0.44) \\
0.28(0.00,0.55)\end{array}$ & $\begin{array}{l}3 \\
1.5\end{array}$ & $\begin{array}{l}14 \\
46\end{array}$ & $\begin{array}{l}19 \\
46\end{array}$ & $\begin{array}{l}30 \\
56\end{array}$ & $\begin{array}{l}5.5 \\
4.9\end{array}$ & $\underset{B}{C}$ \\
\hline Overall $\left(1^{2}=18 \%, P=0.22\right)$ & $\mathcal{L P}=0.006$ & $0.18(0.05,0.31)$ & & & & & & \\
\hline-2.0 & 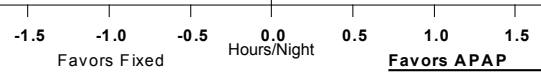 & & & & & & & \\
\hline \multicolumn{9}{|c|}{$\begin{array}{l}\text { Figure } 2 \text { CPAP compliance (hour/night) with APAP versus fixed CPAP: meta-analysis, with subgroup analyses by minimum AHI } \\
\text { threshold. Estimates and } 95 \% \text { Cls by study subgrouped by minimum AHI threshold used in each study. The overall random effects model } \\
\text { meta-analysis is displayed by the black diamond, which spans the width of the } 95 \% \text { CI. Each subgroup meta-analysis, by AHI threshold, is shown } \\
\text { by the open diamonds. Grey boxes are proportional to the weight of each study in the overall meta-analysis. For each meta-analysis the } \mathrm{I}^{2} \\
\text { statistic and the } P \text { value for heterogeneity is displayed. The } P \text { value for the summary estimate is displayed next to the black diamond. Note that } \\
\text { studies favoring APAP are to the right of the vertical } 0 \text { line. Base AHI: baseline apnea-hypopnea index (events/hour) in fixed CPAP group; } \\
\text { compliance fixed: compliance (hour/night) in fixed CPAP group; fixed: fixed CPAP. }\end{array}$} \\
\hline
\end{tabular}

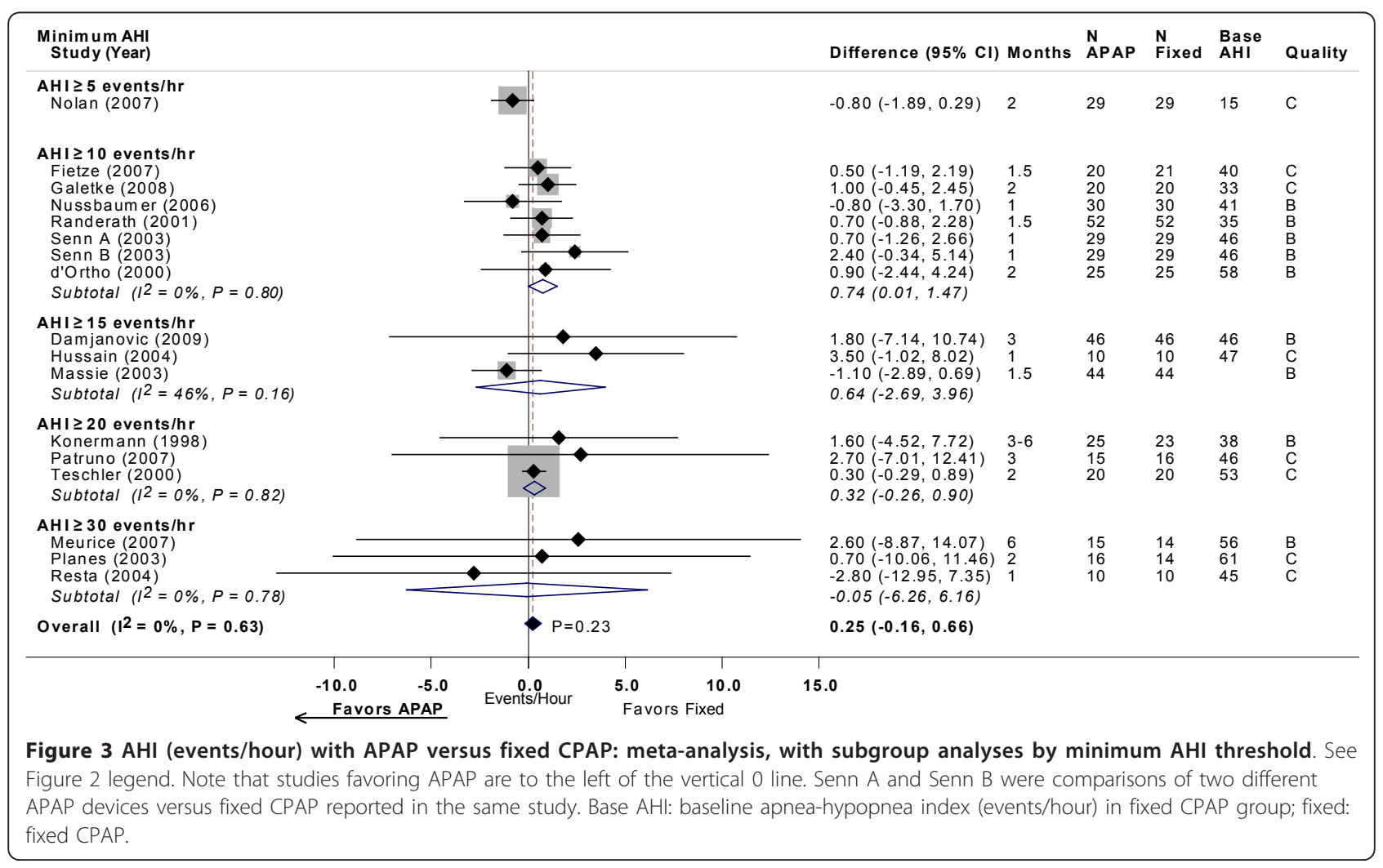




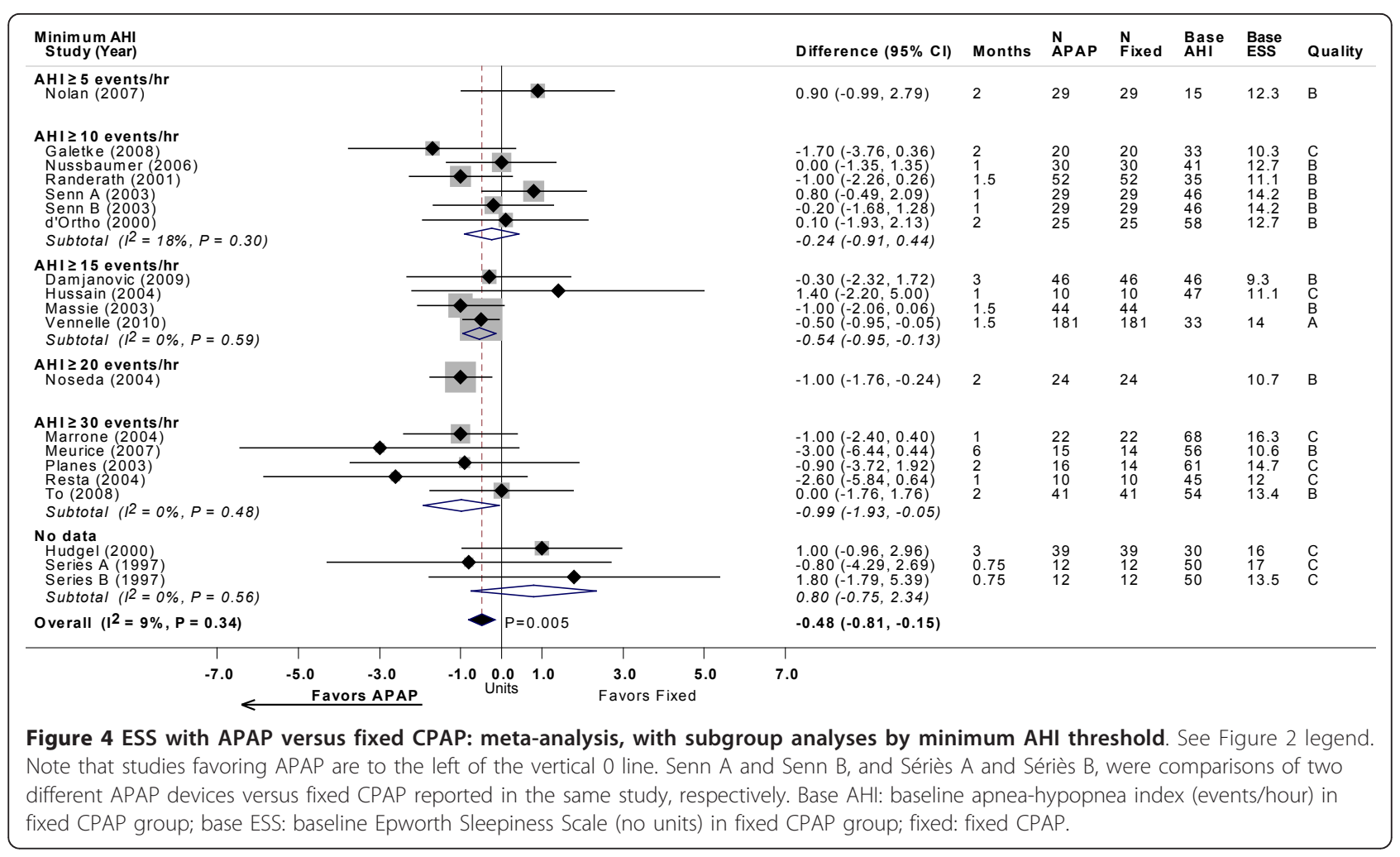

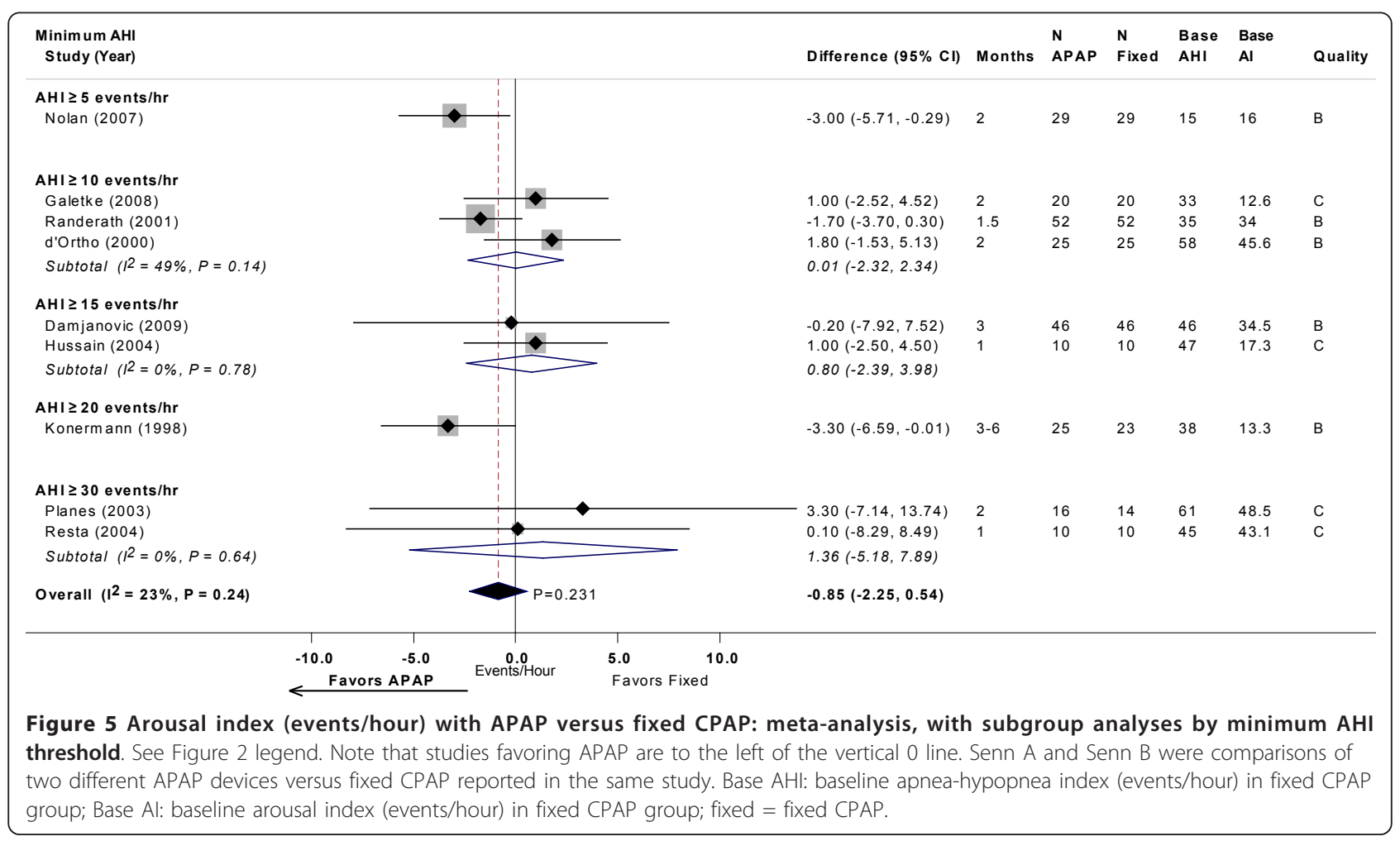




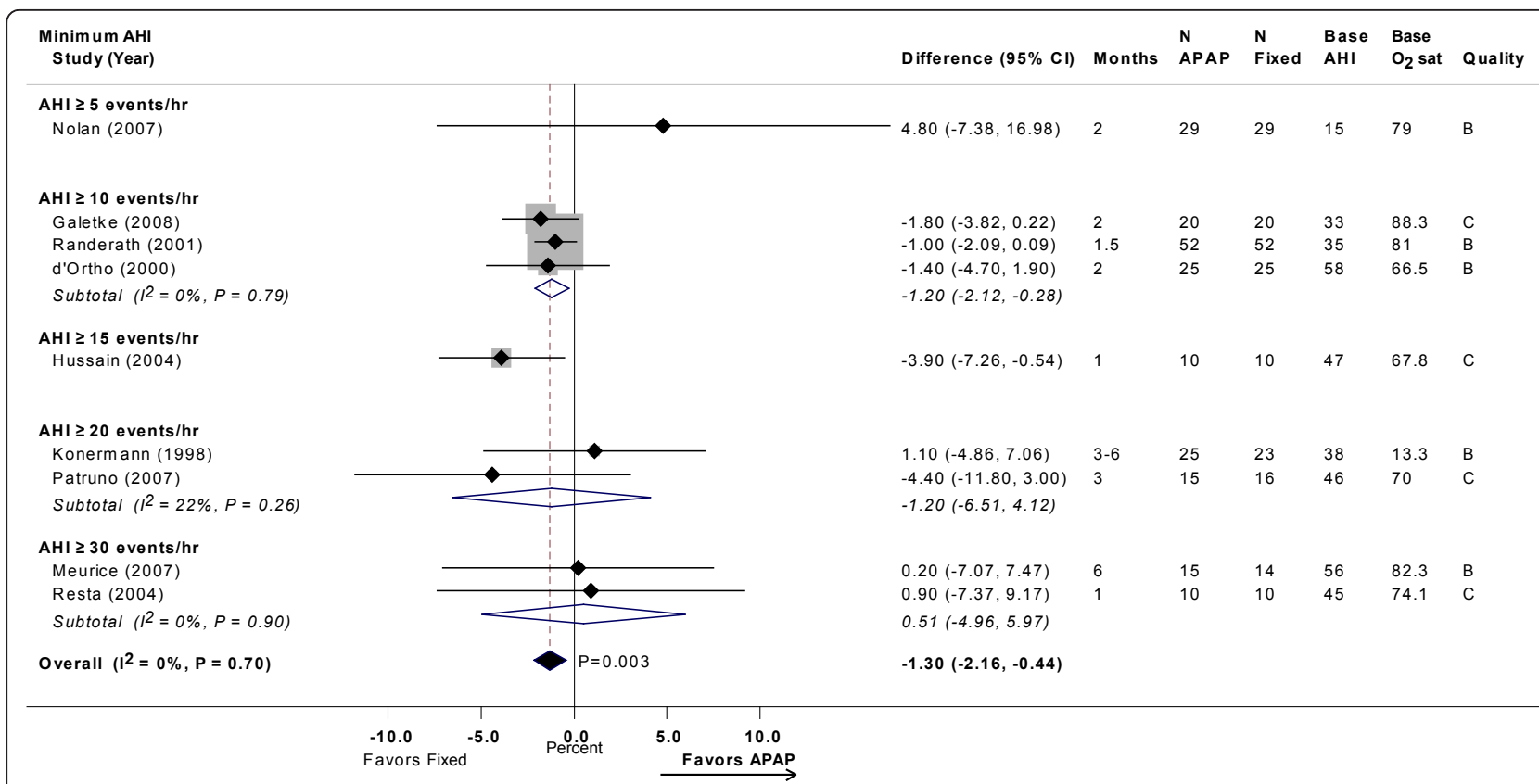

Figure 6 Minimum oxygen saturation (\%) with APAP versus fixed CPAP: meta-analysis, with subgroup analyses by minimum AHI threshold. See Figure 2 legend. Note that studies favoring APAP are to the left of the vertical 0 line. Senn A and Senn B were comparisons of two different APAP devices versus fixed CPAP reported in the same study. Base AHI: baseline apnea-hypopnea index (events/hour) in fixed CPAP group; Base min O2: baseline minimum oxygen saturation (\%) in fixed CPAP group; fixed: fixed CPAP.

significant difference of 11 minutes per night also favoring APAP compared to fixed CPAP. The clinical significance of these reported improvements in ESS and compliance, however, is unclear. The two types of devices were found to result in similar changes from baseline in AHI, quality of life and most other sleep study measures. However, there is also evidence that minimum oxygen saturation improves more with CPAP than with APAP by about $1 \%$. Evidence is limited regarding the relative effect of fixed CPAP and APAP on blood pressure. There were no data on objective clinical outcomes.

The etiology and severity of OSA varies widely across patients, as do patients' symptoms and their ability to tolerate or consistently use treatments. CPAP, specifically, can be cumbersome and uncomfortable to use; therefore, it is of particular importance to identify which subgroups of patients may benefit most from which specific treatments to maximize the effectiveness of intervention. Our subgroup meta-analyses based on different minimum AHI thresholds to define OSA failed to demonstrate any difference in effectiveness between APAP and CPAP for all outcomes. Higher AHI is used as a marker for more severe disease [35] and is associated with greater mortality [1,36-38]; however, our power to find any differences based on baseline AHI was low, particularly since the study eligibility categories overlapped. Even if we had found a difference, it would at best be hypothesis-generating and would need to be confirmed in a primary study. But we found no study that directly analyzed any subgroup of patients who may particularly benefit from a given treatment. It should be noted that experts have opined that APAP may be used in the setting of failed fixed CPAP (CD, personal communication), although we are not aware of a study in such a setting.

Despite the addition of newer studies in our meta-analyses, our findings differed little from those of a Cochrane meta-analysis reported in 2009, which reviewed 30 randomized trials enrolling 1,136 patients in total (the Cochrane review had a larger number of RCTs because it included results from posters and conference proceedings) [4]. The Cochrane review segregated its analysis by study design into cross-over and parallel design studies but did not provide an aggregate analysis combining both types of study design. In that meta-analysis, a statistically significant difference in compliance of 12.6 minutes per night (95\% CI, 4.8 to 21 minutes increase) was found in favor of APAP in crossover studies, but no significant difference in parallel design studies. It also reported a statistically significant decrease in ESS of 0.64 points (95\% CI, 0.12 to 1.16 point decrease) in favor of APAP in cross-over studies, but no significant difference in parallel design studies. We reported similar findings for the two study designs in our full technical review [6]. 
Table 11 Strength of evidence of APAP versus CPAP

\begin{tabular}{|c|c|c|c|c|c|c|}
\hline Outcomes & $\begin{array}{l}\text { Number } \\
\text { of trials }\end{array}$ & $\begin{array}{l}\text { Total } \\
\text { number }\end{array}$ & $\begin{array}{l}\text { Trials with data } \\
\text { for Meta- } \\
\text { analysis }\end{array}$ & $\begin{array}{l}\text { Meta-analysis results } \\
\text { comparing APAP with } \\
\text { CPAP }\end{array}$ & Favors & $\begin{array}{l}\text { Strength } \\
\text { of } \\
\text { evidence }\end{array}$ \\
\hline $\begin{array}{l}\text { Clinical outcomes (death, } \\
\text { cardiovascular events and } \\
\text { others) }\end{array}$ & 0 & 0 & 0 & N/A & & Insufficient \\
\hline Compliance & 24 & 1008 & 22 & $\begin{array}{l}0.18 \text { hours }(95 \% \mathrm{Cl} 0.05 \\
\text { to } 0.31 ; P=0.006)\end{array}$ & APAP & Moderate \\
\hline Apnea-Hypopnea Index & 16 & 548 & 16 & $\begin{array}{l}0.25 \text { events/hour ( } 95 \% \\
\mathrm{Cl}-0.16 \text { to } 0.66 ; \mathrm{NS})\end{array}$ & No difference & Moderate \\
\hline Epworth Sleepiness Scale & 22 & 954 & 18 & $\begin{array}{l}-0.48(95 \% \mathrm{Cl}-0.81 \text { to } \\
-0.15 ; P=0.005)\end{array}$ & APAP & Moderate \\
\hline Arousal Index & 10 & 356 & 9 & $\begin{array}{l}-0.85 \text { events/hour ( } 95 \% \\
\mathrm{Cl}-2.2 \text { to } 0.5 ; \mathrm{NS} \text { ) }\end{array}$ & No difference & Moderate \\
\hline Minimum $\mathrm{O}_{2}$ saturation & 9 & 258 & 9 & $\begin{array}{l}-1.3 \%(95 \% \mathrm{Cl}-2.2 \text { to } \\
-0.4 ; P=0.003)\end{array}$ & CPAP & Moderate \\
\hline Sleep efficiency & 3 & 126 & 0 & & No difference & Insufficient \\
\hline Rapid eye movement sleep & 9 & 273 & 0 & & No difference & Moderate \\
\hline Slow wave sleep & 8 & 243 & 0 & & $\begin{array}{l}\text { No difference in seven trials; one trial } \\
\text { favored APAP }\end{array}$ & Moderate \\
\hline Quality of life & 9 & 509 & 0 & & $\begin{array}{l}\text { No difference in eight trials; one trial } \\
\text { favored APAP }\end{array}$ & Moderate \\
\hline Blood pressure & 3 & 149 & 0 & & $\begin{array}{l}\text { No difference in two trials; one trial } \\
\text { favored CPAP (decrease in diastolic } \\
\text { blood pressure) }\end{array}$ & Insufficient \\
\hline
\end{tabular}

APAP: auto-titrating CPAP; CPAP: continuous positive airway pressure; N/A: not applicable; NS: non-significant.

Follow-up durations in the studies reviewed tended to be short, in the order of weeks to a few months, and are clearly insufficient for the appraisal of the treatment of a life-long disease whose clinical sequelae may take decades to develop. Study dropout rates were also frequently very high, particularly given the short duration of follow-up. In some studies, up to $40 \%$ of participants were lost to follow-up within weeks. The ability to meaningfully interpret the findings from these studies is clearly diminished. Other frequent methodological problems included incomplete reporting and/or inadequate analyses. In particular, relatively few studies provided the net differences between interventions (in parallel design studies) or the difference between final values with appropriate adjustments for correlation (in cross-over studies) with their confidence intervals and $P$ values. Thus for the large majority of studies, we had to estimate the confidence intervals of the differences between interventions. We also did not search for unpublished and non-English language studies.

\section{Conclusions}

APAP and CPAP were similar in affecting relatively short-term changes in AHI, quality of life, and most other sleep study measures in the treatment of patients with moderate to severe OSA but without significant comorbidities. APAP, however, did reduce sleepiness by approximately 0.5 ESS points more than fixed CPAP. Patients who received APAP also had objectively measured compliance of 11 minutes per night more than those who received fixed CPAP. We surmise that the clinical significance of these reported improvements in ESS and compliance is marginal at best. It is doubtful that additional short-term trials comparing APAP and CPAP to examine these measures will substantially alter these results. However, longer-term and larger trials that evaluate clinical outcomes, such as cardiovascular events, and directly estimate differential effects in different sub-populations may be of value. Furthermore, the current trial evidence is limited to patients newly diagnosed with sleep apnea or who are otherwise naïve to CPAP; thus, future trials of patients who had previously used CPAP may be of value. For now, based on the available data from experimental studies on short-term effects only, the decision to use APAP versus CPAP may well depend on individual patient preferences, specific reasons for non-compliance, costs and other practical considerations that clinicians and patients will need to assess on an individual basis.

\section{Abbreviations}

AHI: apnea-hypopnea index; AHRQ: Agency for Healthcare Research and Quality; APAP: auto-titrating positive airway pressure; CPAP: fixed continuous positive airway pressure; ESS: Epworth Sleepiness Scale; OSA: obstructive sleep apnea; RCT: randomized controlled trial; REM: rapid eye movement; SF$36^{\circledR}{ }^{\circledR}$ : Short Form Health Survey. 


\section{Acknowledgements}

This research was funded by the AHRQ, US Department of Health and Human Services (Contract No. 290200710055 I). AHRQ participated in formulating the study questions but did not participate in the literature search; determination of study eligibility criteria; data analysis or interpretation; or preparation, review, or approval of the manuscript for publication.

\section{Author details}

'Institute of Clinical Research and Health Policy Studies, Tufts University School of Medicine, Tufts Medical Center, Boston, MA, USA. ${ }^{2}$ Center for Sleep Medicine, Tufts Medical Center, Boston, MA, USA.

\section{Authors' contributions}

$\mathrm{SI}$ and EMB designed the study, extracted and analyzed the data and drafted the manuscript. CD provided domain expert input, and reviewed and edited the manuscript. KP, NO, GDK and MC extracted and analyzed the study data, and also reviewed and edited the manuscript. All authors read and approved the final manuscript.

\section{Competing interests}

The authors declare that they have no competing interests.

Received: 11 November 2011 Accepted: 8 March 2012

Published: 8 March 2012

\section{References}

1. Young T, Finn L, Peppard PE, Szklo-Coxe M, Austin D, Nieto FJ, Stubbs R, Hla KM: Sleep disordered breathing and mortality: eighteen-year followup of the Wisconsin sleep cohort. Sleep 2008, 31:1071-1078.

2. Hiestand DM, Britz P, Goldman M, Phillips B: Prevalence of symptoms and risk of sleep apnea in the US population: results from the national sleep foundation sleep in America 2005 poll. Chest 2006, 130:780-786.

3. Veasey SC, Guilleminault C, Strohl KP, Sanders MH, Ballard RD, Magalang UJ: Medical therapy for obstructive sleep apnea: a review by the Medical Therapy for Obstructive Sleep Apnea Task Force of the Standards of Practice Committee of the American Academy of Sleep Medicine. Sleep 2006, 29:1036-1044.

4. Smith I, Lasserson TJ: Pressure modification for improving usage of continuous positive airway pressure machines in adults with obstructive sleep apnoea. Cochrane Database Syst Rev 2009, , 4: CD003531, Update of Cochrane Database Syst Rev 2004, (4):CD003531.

5. Agency for Healthcare Research and Quality. Methods Reference Guide for Effectiveness and Comparative Effectiveness Reviews, Version 1.0 Rockville, MD: Agency for Healthcare Research and Quality; 2010.

6. Balk EM, Moorthy D, Obadan NO, Patel K, Ip S, Chung M, Bannuru RR, Kitsios GD, Sen S, lovin RC, Gaylor JM, D'Ambrosio C, Lau J: Diagnosis and treatment of obstructive sleep apnea in adults. AHRQ Comparative Effectiveness Reviews Rockville, MD: Agency for Healthcare Research and Quality; 2011, Report No.: 11-EHC052.

7. Wallace BC, Trikalinos TA, Lau J, Brodley C, Schmid CH: Semi-automated screening of biomedical citations for systematic reviews. BMC Bioinformatics 2010, 11:55.

8. DerSimonian R, Laird N: Meta-analysis in clinical trials. Control Clin Trials 1986, 7:177-188

9. Higgins JP, Thompson SG, Deeks JJ, Altman DG: Measuring inconsistency in meta-analyses. BMJ 2003, 327:557-560

10. Damjanovic D, Fluck A, Bremer H, Muller-Quernheim J, Idzko M, Sorichter S: Compliance in sleep apnoea therapy: influence of home care support and pressure mode. Eur Respir J 2009, 33:804-811.

11. Fietze I, Glos M, Moebus I, Witt C, Penzel T, Baumann G: Automatic pressure titration with APAP is as effective as manual titration with CPAP in patients with obstructive sleep apnea. Respiration 2007, 74:279-286.

12. Galetke W, Anduleit N, Richter K, Stieglitz S, Randerath WJ: Comparison of automatic and continuous positive airway pressure in a night-by-night analysis: a randomized, crossover study. Respiration 2008, 75:163-169.

13. Meurice JC, Cornette A, Philip-Joet F, Pepin JL, Escourrou P, Ingrand P, Veale D, ANTADIR "PPC" Working Group: Evaluation of autoCPAP devices in home treatment of sleep apnea/hypopnea syndrome. Sleep Med 2007, 8:695-703.
14. Nolan GM, Doherty LS, Mc Nicholas WT: Auto-adjusting versus fixed positive pressure therapy in mild to moderate obstructive sleep apnoea. Sleep 2007, 30:189-194.

15. Nussbaumer Y, Bloch KE, Genser T, Thurnheer R: Equivalence of autoadjusted and constant continuous positive airway pressure in home treatment of sleep apnea. Chest 2006, 129:638-643.

16. Patruno V, Aiolfi S, Costantino G, Murgia R, Selmi C, Malliani A, Montano N: Fixed and autoadjusting continuous positive airway pressure treatments are not similar in reducing cardiovascular risk factors in patients with obstructive sleep apnea. Chest 2007, 131:1393-1399.

17. To KW, Chan WC, Choo KL, Lam WK, Wong KK, Hui DS: A randomized cross-over study of auto-continuous positive airway pressure versus fixed-continuous positive airway pressure in patients with obstructive sleep apnoea. Respirology 2008, 13:79-86.

18. Hudgel DW, Fung C: A long-term randomized, cross-over comparison of auto-titrating and standard nasal continuous airway pressure. Sleep 2000, 23:645-648.

19. Hukins C: Comparative study of autotitrating and fixed-pressure CPAP in the home: a randomized, single-blind crossover trial. Sleep 2004, 27:1512-1517.

20. Hussain SF, Love L, Burt H, Fleetham JA: A randomized trial of autotitrating CPAP and fixed CPAP in the treatment of obstructive sleep apnea-hypopnea. Respiratory Med 2004, 98:330-333.

21. Marrone O, Resta O, Salvaggio A, Giliberti T, Stefano A, Insalaco G: Preference for fixed or automatic CPAP in patients with obstructive sleep apnea syndrome. Sleep Med 2004, 5:247-251.

22. Massie CA, McArdle N, Hart RW, Schmidt-Nowara WW, Lankford A Hudgel DW, Gordon N, Douglas NJ: Comparison between automatic and fixed positive airway pressure therapy in the home. Am J Respir Crit Care Med 2003, 167:20-23.

23. Noseda A, Kempenaers C, Kerkhofs M, Braun S, Linkowski P, Jann E: Constant vs auto-continuous positive airway pressure in patients with sleep apnea hypopnea syndrome and a high variability in pressure requirement. Chest 2004, 126:31-37.

24. Resta O, Carratu P, Depalo A, Giliberti T, Ardito M, Marrone O, Insalaco G: Effects of fixed compared to automatic CPAP on sleep in Obstructive Sleep Apnoea Syndrome. Monaldi Arch Chest Dis 2004, 61:153-156.

25. Senn O, Brack T, Matthews F, Russi EW, Bloch KE: Randomized short-term trial of two autoCPAP devices versus fixed continuous positive airway pressure for the treatment of sleep apnea. Am J Respir Crit Care Med 2003, 168:1506-1511.

26. Randerath WJ, Schraeder O, Galetke W, Feldmeyer F, Ruhle KH: Autoadjusting CPAP therapy based on impedance efficacy, compliance and acceptance. Am J Respir Crit Care Med 2001, 163:652-657.

27. Series F, Marc I: Efficacy of automatic continuous positive airway pressure therapy that uses an estimated required pressure in the treatment of the obstructive sleep apnea syndrome. Ann Intern Med 1997, 127:588-595.

28. Teschler $\mathrm{H}$, Wessendorf $T E$, Farhat $A A$, Konietzko $N$, Berthon-Jones $M$ : Two months auto-adjusting versus conventional nCPAP for obstructive sleep apnoea syndrome. Eur Respir J 2000, 15:990-995.

29. Planes C, d'Ortho MP, Foucher A, Berkani M, Leroux K, Essalhi M, Delclaux C, Quera-Salva MA, Lofaso F: Efficacy and cost of home-initiated auto-nCPAP versus conventional nCPAP. Sleep 2003, 26:156-160.

30. Vennelle M, White S, Riha RL, Mackay TW, Engleman HM, Douglas NJ: Randomized controlled trial of variable-pressure versus fixed-pressure continuous positive airway pressure (CPAP) treatment for patients with obstructive sleep apnea/hypopnea syndrome (OSAHS). Sleep 2010, 33:267-271.

31. Konermann M, Sanner BM, Vyleta M, Laschewski F, Groetz J, Sturm A, Zidek W: Use of conventional and self-adjusting nasal continuous positive airway pressure for treatment of severe obstructive sleep apnea syndrome: a comparative study. Chest 1998, 113:714-718.

32. d'Ortho MP, Grillier-Lanoir V, Levy P, Goldenberg F, Corriger E, Harf A, Lofaso F: Constant vs. automatic continuous positive airway pressure therapy: home evaluation. Chest 2000, 118:1010-1017.

33. West SD, Jones DR, Stradling JR: Comparison of three ways to determine and deliver pressure during nasal CPAP therapy for obstructive sleep apnoea. Thorax 2006, 61:226-231.

34. Ayas NT, Patel SR, Malhotra A, Schulzer M, Malhotra M, Jung D, Fleetham J, White DP: Auto-titrating versus standard continuous positive airway pressure for the treatment of obstructive sleep apnea: results of a metaanalysis. Sleep 2004, 27:249-253. 
35. American Academy of Sleep Medicine: The International Classification of Sleep Disorders, 2nd Edition: Diagnostic and Coding Manual Westchester, LL: American Academy of Sleep Medicine; 2005.

36. Lavie $\mathrm{P}$, Lavie L, Herer P: All-cause mortality in males with sleep apnoea syndrome: declining mortality rates with age. Eur Respir J 2005, 25:514-520.

37. Punjabi NM, Caffo BS, Goodwin JL, Gottlieb DJ, Newman AB, O'Connor GT, Rapoport DM, Redline S, Resnick HE, Robbins JA, Shahar E, Unruh ML, Samet JM: Sleep-disordered breathing and mortality: a prospective cohort study. PLoS Med 2009, 6(8):e1000132.

38. Lavie P, Here P, Peled R, Berger I, Yoffe N, Zomer J, Rubin AH: Mortality in sleep apnea patients: a multivariate analysis of risk factors. Sleep 1995, 18:149-157.

doi:10.1186/2046-4053-1-20

Cite this article as: Ip et al:: Auto-titrating versus fixed continuous

positive airway pressure for the treatment of obstructive sleep apnea: a systematic review with meta-analyses. Systematic Reviews 2012 1:20.

\section{Submit your next manuscript to BioMed Central} and take full advantage of:

- Convenient online submission

- Thorough peer review

- No space constraints or color figure charges

- Immediate publication on acceptance

- Inclusion in PubMed, CAS, Scopus and Google Scholar

- Research which is freely available for redistribution

Submit your manuscript at www.biomedcentral.com/submit
C Biomed Central 